\title{
Trajectory Optimization for Target Localization Using Small Unmanned Aerial Vehicles
}

\author{
Sameera S. Ponda*, Richard M. Kolacinski ${ }^{\dagger}$ and Emilio Frazzoli ${ }^{\ddagger}$
}

\begin{abstract}
Small unmanned aerial vehicles (UAVs) equipped with navigation and video capabilities can be used to perform target localization. Combining UAV state estimates with image data leads to bearing measurements of the target that can be processed to determine its position. This 3-D bearings-only estimation problem is nonlinear and traditional filtering methods are prone to biases, noisy estimates, and filter instabilities. The performance of the target localization is highly dependent on the vehicle trajectory, motivating the development of optimal UAV trajectories. This work presents methods for designing trajectories that increase the amount of information provided by the measurements and shows that these trajectories lead to enhanced estimation performance.

The Fisher Information Matrix (FIM) is used to quantify the information provided by the measurements. Several objective functions based on the FIM are considered and the A-optimality criterion is shown to be the best suited for trajectory optimization in the 3-D bearings-only target localization problem. The resulting trajectories produce spirals, which increase the angular separation between measurements and reduce the range to the target, supporting geometric intuition. The problem of simultaneous target estimation and vehicle trajectory optimization is explored and the resulting algorithms produce vehicle trajectories that increase the information provided by the measurements, enhancing the target estimation performance by increasing accuracy, reducing uncertainty and improving filter convergence.
\end{abstract}

\section{Introduction}

\section{I.A. Motivation}

Recent advances in technology are encouraging the use of small unmanned aerial vehicles (UAVs) for intelligence, reconnaissance, and surveillance (ISR) missions. Equipped with onboard electro-optical and infrared cameras, as well as navigation and communications hardware, current small UAVs are capable of surveying the environment, providing the operator with real-time visual information rapidly, inexpensively and with low risk to human life. Figure 1 shows a few examples of small UAVs, such as Raven and Wasp, which are currently used by the U.S. Army for many military missions overseas.

A particularly interesting ISR mission for small UAVs is to localize and track targets detected by onboard sensors. Using imagery data of a target from different UAV locations, with knowledge of the UAV position and orientation at the time of the measurements, an estimate of the target location can be computed through triangulation (Figure 2). This capability is useful in environments for which no a priori information is available. In addition to ISR missions, this localization capability would be invaluable for several other applications, including traffic monitoring, forest fire localization, scientific surveys in dangerous conditions, border and harbor patrol, search and rescue, and wildlife tracking.

The main challenges involved in target localization include maintaining the target in the field of view, and developing a UAV trajectory such that the target location estimation error is minimized. For a human operator, maintaining the target in the field of view is a challenging task, due to limited vehicle maneuverability, a noisy environment (mainly due to wind gusts) and an overall lack of proprioceptive feedback provided to

\footnotetext{
${ }^{*}$ Ph.D. candidate, Department of Aeronautics and Astronautics, Massachusetts Institute of Technology, Cambridge, MA 02139

${ }^{\dagger}$ Principal Member of Technical Staff, The Charles Stark Draper Laboratory, 555 Technology Square, Cambridge, MA 02139

$¥$ Associate Professor of Aeronautics and Astronautics, Department of Aeronautics and Astronautics, Massachusetts Institute of Technology, Cambridge, MA 02139
} 


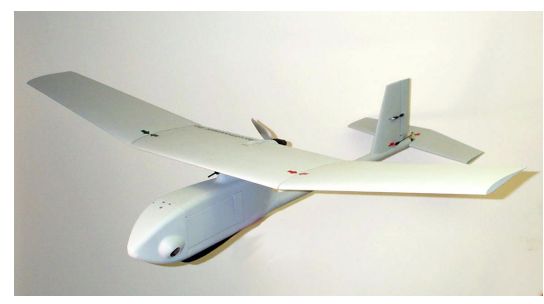

(a) Raven (AeroVironment, Inc)

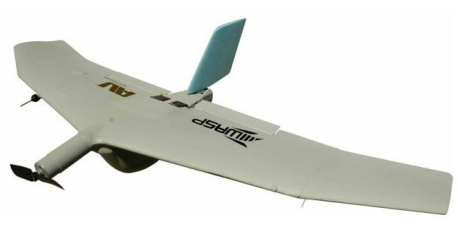

(b) Wasp Block III (AeroVironment, Inc)

Figure 1: Examples of small unmanned aerial systems

the operator from the vehicle. It is also difficult for a human operator to have an intuitive understanding of how best to fly the vehicle in such a way that the target location estimation error is minimized. For stationary target tracking, circular trajectories overhead the target are widely accepted in the literature as good trajectories. ${ }^{5,38,46}$ However, the optimal circling radius is not known and, if the target is moving, the operator may not deduce the best vehicle trajectory. Autonomous flight path generation is therefore essential to successful target localization. The purposes of this work are to provide methods for designing UAV trajectories that enable accurate vision-based target localization and to show that these trajectories lead to enhanced localization performance.

\section{I.B. Problem Description}

This section describes the problem of target localization using UAVs equipped with vision-based sensors. Visual measurements of the target can be transformed into bearing measurements using the vehicle position and orientation, the camera orientation with respect to the vehicle, and the pixel location of the target. For the purpose of this project, image processing for target recognition is assumed and the pixel location corresponding to the centroid of the target is available. Using successive bearing measurements the target position can be computed through triangulation (Figure 2).

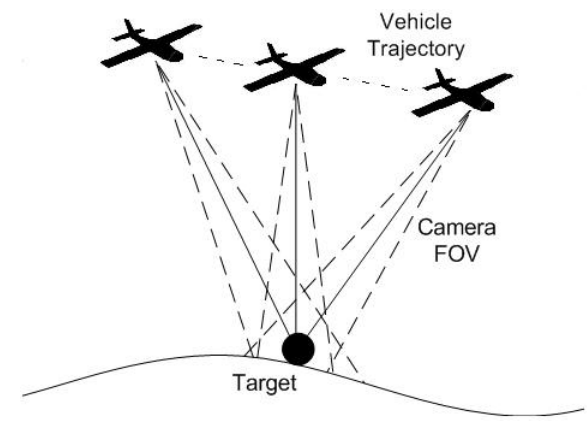

Figure 2: Illustration of target localization using a UAV with vision

The task of target localization presents several challenges. Most modern small UAVs are equipped with GPS and inertial sensors, but are designed to be inexpensive and therefore include low quality sensors and poor navigation algorithms, leading to inaccurate vehicle state estimation. Typical navigation errors for small UAVs involve standard deviations of about 13 feet for position accuracy using GPS, 10 degrees for attitude using gyros (roll and pitch), and 5 degrees for heading using a magnetometer or electronic compass. Furthermore, in urban settings, magnetometer readings can be severely affected by the surrounding magnetic fields, resulting in much larger heading errors. Given these large navigation errors it is difficult to achieve a high level of precision in target estimation, since errors in the vehicle state propagate into the targeting algorithms. However, the geometry of the problem can be exploited to determine the optimal measurement locations, leading to the development of vehicle trajectories that reduce the target localization error. Other elements that affect flight path generation, such as vehicle constraints, sensor constraints, computational limitations and mission requirements, should also be considered. Target dynamics, environmental constraints, and disturbances must be taken into account as well, and the varying nature of these suggests that the solution should be updated in real time. The following sections provide more detail about the problem considered in this project, define the scope, and state the major underlying assumptions. 


\section{I.B.1. Platform Vehicle and Target Types}

The platform vehicle for this project is restricted to a small fixed-wing airplane equipped with an onboard navigation system, imaging sensors, controllable actuators (elevator and rudder), and basic onboard processing and communication capabilities. An example is the Raven, made by AeroVironment, Inc (Figure 1). Small fixed-wing UAVs are limited in their operating range and controllability. They typically operate at low altitudes (between 100 to $500 \mathrm{ft}$ above ground level). The lower limit is due to communication restrictions (obstructions and increased noise from ground reflections make communications between the UAV and the ground station difficult below $100 \mathrm{ft}$ ). Even though the upper limit of operation is usually higher, the image quality degrades above $500 \mathrm{ft},{ }^{36}$ providing an upper bound on altitude for vision-based UAV operations. Additionally, small UAVs typically fly at low uniform speeds (the Raven cruise velocity is $30 \mathrm{mph}$ ). For this project the UAV is assumed to have constant altitude, constant velocity and a maximum turn rate constraint.

The targets of most interest within the scope of the project include small stationary ground targets and small slow-moving ground targets. Given the operating range of the UAV, the useful imagery data obtained involves mostly these kinds of targets. For the purpose of this work no prior motion models are assumed and moving targets are treated as stochastic. If further information is available, such as road maps or target classifications, motion models could be incorporated to improve estimation accuracy and convergence, however this is beyond the current scope of the paper.

\section{I.B.2. System Dynamics and Measurement Model}

The UAV is assumed to have six degrees of freedom $(6 \mathrm{DOF}), 3$ translational and 3 rotational. The target position is estimated in three-dimensional space. In order to obtain a measurement from an image it is necessary to have: the UAV position and orientation, the camera pointing angle with respect to the UAV, and the pixel location of the target. The camera pointing angle with respect to the UAV is typically well known and can be either fixed or controllable for a camera mounted on gimbals. The vehicle position and orientation are taken from the UAV navigation system and usually have large errors that propagate into the target location estimation.

The pixel location of the target can be transformed into two bearing angles $\left(\alpha_{1}\right.$ and $\left.\alpha_{2}\right)$ from the camera pointing axis to a vector that passes through the target and through the camera focal point (Figure 3). Using a series of rotations (from the camera frame to the UAV body frame, and from the UAV body frame

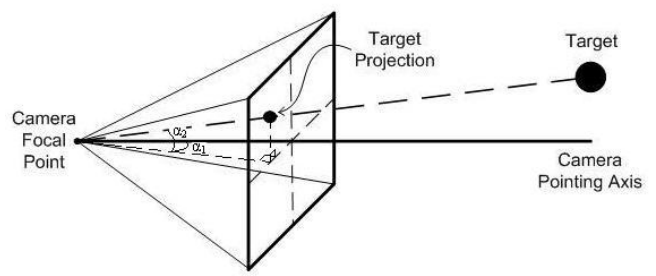

Figure 3: Measurement process

to the earth relative frame), the two angles $\alpha_{1}$ and $\alpha_{2}$ can be transformed into overall azimuth and elevation angles $(\beta$ and $\phi)$ which define a bearing between the target and the UAV (Figure 4). The measurements model is given by,

$$
\begin{aligned}
& \beta=\tan ^{-1}\left(\frac{r_{x}}{r_{y}}\right) \\
& \phi=\tan ^{-1}\left(\frac{r_{z}}{\sqrt{r_{x}^{2}+r_{y}^{2}}}\right)
\end{aligned}
$$

It is important to note that image-based measurements only provide bearing information to the target and not range. The magnitude of the relative vector, $|\mathbf{r}|$, therefore cannot be determined using only one measurement. Two or more measurements are needed to obtain an estimate of the target location. For the purpose of this project a gimballed camera system is assumed to ensure that the target remains in the field of view of the camera. 


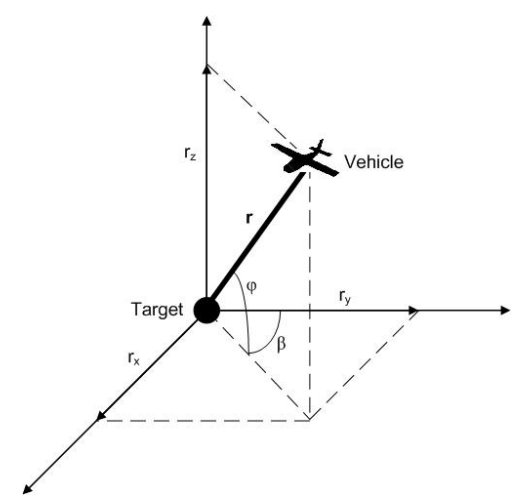

Figure 4: Azimuth $(\beta)$ and elevation $(\phi)$ between vehicle and target

\section{I.B.3. Error Propagation}

It is necessary to understand how errors in the vehicle state estimate propagate through to the target location estimate, to exploit this geometry and produce trajectories that minimize the target localization error. Figure 5 shows the effects of vehicle position and orientation errors on the combined target localization error. Orthogonal measurements produce a lower combined error than non-orthogonal measurements for both position and orientation errors. Furthermore, for vehicle orientation errors, a smaller range greatly reduces the combined target localization error. Therefore the geometric intuition suggests that to reduce target localization error it is desirable to increase the angular separation between the measurements and to minimize the relative range to the target.

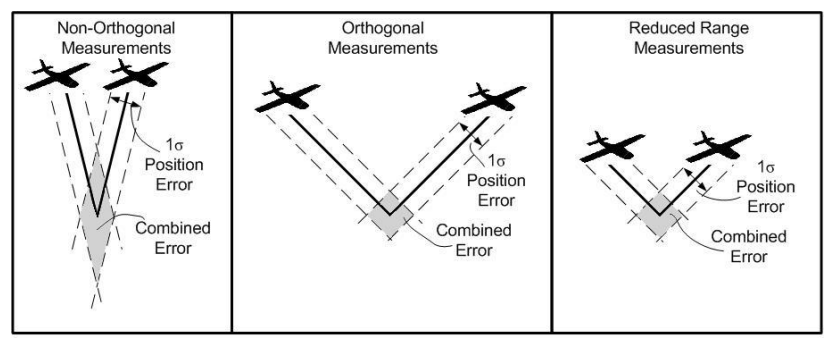

(a) Vehicle position errors

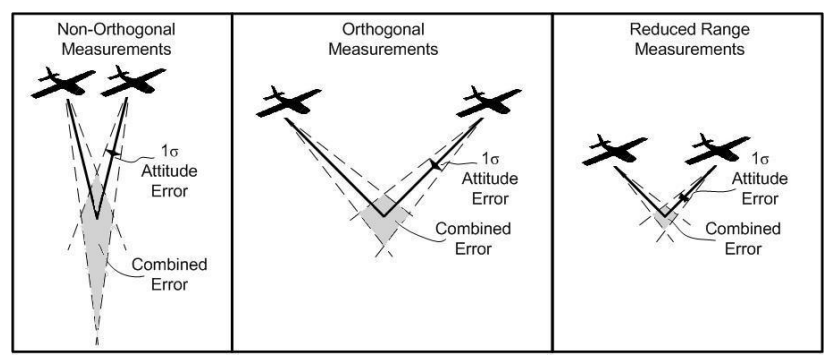

(b) Vehicle orientation errors

Figure 5: Effect of measurement orthogonality and range on target localization

While analysis of geometric trends shows the underlying importance of selecting a good flight path, it does not provide a proper framework for designing such a path. The approach taken in this paper is to use the Fisher Information Matrix to provide a framework for quantifying the information provided by a set of measurements. Positioning the vehicle to maximize this information content leads to the design of flight trajectories that minimize the target localization error. 


\section{I.C. Literature Review}

The problem of trajectory optimization for vision-based target localization has several related areas of research. Target localization and tracking has been studied extensively in numerous texts. ${ }^{4,39}$ For bearingsonly target localization, several important considerations for filter development and observability requirements have been explored in the context of passive sonar applications. Other relevant research areas for this project involve information-based sensor placement techniques for optimal parameter estimation and UAV operations for vision-based target localization. This section provides details that are particularly relevant to the problem of trajectory optimization for vision-based target localization.

The problem of bearings-only target tracking has a rich history in the context of passive sonar applications for underwater vehicles dating back to the 1960's. The focus is mainly on analytical derivations for the observability criteria of the estimation process, and comparisons of the convergence properties and performance of the different types of filters used for passive target localization. Lindgren et al. ${ }^{26}$ and Nardone et al. ${ }^{32}$ illustrate the importance of vehicle maneuvers for proper estimation observability in 2-D bearings-only estimation. Later work by Hammel, ${ }^{15}$ Levine, ${ }^{25}$ Fogel, ${ }^{11}$ and Becker ${ }^{6}$ extend the observability analysis to higher dimensional cases. Several filter designs for bearings-only target estimation have been proposed including least-squares ${ }^{26}$ maximum likelihood estimation ${ }^{9,41}$ and Kalman filtering. ${ }^{2,31}$ Traditional Extended Kalman Filtering approaches are shown to be sensitive to initialization and measurement errors, causing premature covariance collapse and other filter instabilities. ${ }^{2}$ Pseudolinear filter formulations and modified polar coordinate filters are proposed by Aidala et al., ${ }^{1,3}$ attempting to improve filter convergence and remove biases, and comparisons of performance for different filtering algorithms are explored by Nardone et al. ${ }^{31}$

Given the strong dependence of estimation performance on the vehicle's maneuvers, subsequent research of relevance focuses mainly on developing methods for optimizing the vehicle trajectory to achieve the best target estimation performance. A common approach is to use objective functions derived from the Fisher Information Matrix (FIM). The inverse of the FIM is referred to in the literature as the Cramér-Rao Lower Bound (CRLB) and provides a lower bound on the covariance of the estimation for any unbiased estimator. Past research on vehicle trajectory optimization by Le Cadre et al. ${ }^{21-23}$ uses the determinant of the FIM to optimize vehicle trajectories. Of note is that the determinant of the FIM does not satisfy the Additive Monotonicity Property, therefore one point optimization does not necessarily provide the optimal answer for the whole sequence of controls. ${ }^{21}$ Oshman and Davidson ${ }^{33}$ also use the determinant of the FIM to show enhanced target estimation. Other optimization functions include the standard deviation value for the range, ${ }^{10}$ the trace of the CRLB, ${ }^{16,17}$ and the determinant of the error covariance matrix. ${ }^{13,28}$ Other optimization approaches considered by $\mathrm{Liu}^{27}$ and by Passerieux and Van Cappel ${ }^{35}$ involve classical optimal control techniques, however, these results do not easily scale to more complex target motions or higher order dynamics.

Although most of the previous research in bearings-only tracking and vehicle trajectory optimization was within a passive sonar context, recent attention has been given to the problem of vision-based target localization using UAVs. Ivey and Johnson ${ }^{18}$ propose a vision-based target tracking filter for use on board a UAV, comparing both an Extended Kalman Filter (EKF) and a Square-Root Unscented Kalman Filter (SRUKF). Barber et al. ${ }^{5}$ explore stationary target localization from small fixed-wing UAVs using a Recursive Least Squares (RLS) filter. They also explore flight path optimization by finding an optimal altitude and radius for a circular trajectory above the stationary target. Rafi et al. ${ }^{38}$ also consider radius optimization for circular trajectories of fixed altitude for tracking stationary and constant velocity targets. Quigley et al. ${ }^{37}$ propose Hopf bifurcation techniques for trajectory optimization that result in spirals which converge to a constant radius circle (altitude is assumed constant). These results for stationary targets rely on limit cycle behavior, and although an ad-hoc approach at trajectory planning is taken, the results capture the geometric intuition of the bearings-only target tracking problem. Frew ${ }^{13}$ explores the problem of trajectory planning for a 2-D ground robot equipped with vision sensors, using the determinant of the error covariance matrix as the objective function. Kuang et al. ${ }^{20}$ implement maneuver optimization techniques that minimize the error estimation in a least squares sense. The problem of coordinated flight for multiple UAVs has also been studied. Frew et al. ${ }^{12}$ design trajectories for a network of UAVs for radio source localization using an information-theoretic approach. Bethke et al. ${ }^{7}$ consider the use of multiple quad-rotor UAVs for cooperative target tracking using a RLS approach. Watanabe et al. ${ }^{45}$ consider a formation of two airplanes circling a stationary target, optimizing the trajectories by minimizing the variance of the target position estimate. Whitacre and Campbell ${ }^{46}$ also explore the problem of designing coordinated periodic orbits for two UAVs circling a target. Wise and Rysdyk ${ }^{47}$ show coordinated flight for two UAVs tracking a moving target in windy 
conditions, and Ousingsawat and Campbell ${ }^{34}$ use the determinant of the FIM and receding horizon control techniques to design trajectories for multiple vehicles to track stationary, moving and multiple targets.

Another important area of research involves exploring information-theoretic sensor placement techniques for enhancing parameter estimation. Uciński ${ }^{44}$ provides an excellent review of the state-of-the-art for optimal measurement methods for distributed parameter system identification. Several sensor placement methods involve optimizing over cost functions based on the FIM to maximize the information content provided by a set of measurements. A main issue with this approach is that the calculation of the FIM involves the parameter to be estimated, which, by virtue of the problem statement, is an unknown quantity. If the estimation and sensor placement problems are combined, the dependence of sensor optimization on the parameters to be estimated creates a complex nonlinear problem which is difficult to solve using classical techniques. Another issue is deciding on a scalar cost function based on the FIM which captures the properties of interest. Uciński ${ }^{44}$ gives several examples of past research in sensor placement and describes the advantages and disadvantages of different optimality criteria. Grocholsky et al. ${ }^{14}$ consider sensor trajectory design for multiple sensor platforms for bearings-only target tracking. Sinha et al. ${ }^{40}$ study the problem of coordinating the placement of UAVs equipped with Ground Moving Target Indicators (GMTI) for tracking ground targets. Martínez and Bullo ${ }^{30}$ focus on sensor placement techniques for target tracking using range based measurements by computing the determinant of the FIM and providing an analytic derivation of the cost function. Mandic and Frazzoli ${ }^{29}$ study the placement of ground sensors for the localization of a sniper using acoustic (range-based) measurements. Kaplan and Cevher ${ }^{19}$ study sensor management for fusing bearings-only sensors with different constraints and capabilities using an information-theoretic approach.

This work builds upon previous literature by developing an information-theoretic framework for designing UAV trajectories to localize targets using bearings-only measurements. Several matrix norms for the FIM are considered and the trace of the inverse of the FIM is chosen as the best suited optimality criterion for 3 -D bearings-only target localization. The problem of simultaneous target estimation and vehicle trajectory optimization is explored and the resulting algorithms are shown to produce vehicle trajectories that increase the information provided by the measurements, enhancing the target estimation performance by increasing accuracy, reducing uncertainty and improving filter convergence.

\section{Target Location Estimation}

The problem of target localization and tracking using bearings-only measurements is a difficult task involving a nonlinear measurement process, which, when linearized, can lead to time-varying parameters, biases and in some cases premature filter divergence. ${ }^{2}$ The most common estimation algorithms used for bearings-only target localization are: Least Squares (batch and recursive forms), Maximum Likelihood Estimator, Extended Kalman Filter (EKF), and Particle Filters or Bayesian Methods. This section introduces the Fisher Information Matrix and Cramér-Rao Lower Bound as tools to measure estimation performance. It also presents an EKF for performing target localization using bearings-only measurements.

\section{II.A. Cramér-Rao Lower Bound and the Fisher Information Matrix}

A common measure of performance for an estimator is its error covariance, which represents the uncertainty associated with the accuracy of the estimation results. The covariance should ideally be as low as possible but can only be lowered up to a certain threshold defined by the Cramér-Rao Lower Bound (CRLB). The CRLB is based on the physical properties of the system and the geometry associated with the estimation problem and provides a tight lower bound on the achievable covariance of the estimator. The performance of any unbiased estimator can be quantified by comparing its covariance to the Cramér-Rao Lower Bound. Estimators that achieve this lower bound are called efficient. It is important to note that the CRLB is not a function of the estimation method but of the geometry of the problem; therefore, it provides a limit on the lowest uncertainty that can be obtained for a particular system using a specific set of measurements. Its inverse is referred to as the Fisher Information Matrix (FIM) and provides a measure of the amount of information contained in a given set of measurements about the estimation process. For a review of the CRLB and the Fisher Information Matrix refer to Lehmann, ${ }^{24}$ Ristic $^{39}$ and Uciński. ${ }^{44}$

For the target tracking problem, the estimation error covariance is lower bounded by the inverse of the Fisher Information Matrix,

$$
\mathbf{P}_{k \mid k}=\mathbb{E}\left\{\left[\hat{\mathbf{x}}_{k \mid k}-\mathbf{x}_{k}\right]\left[\hat{\mathbf{x}}_{k \mid k}-\mathbf{x}_{k}\right]^{T}\right\} \geq \mathbf{J}_{k}^{-1}
$$


where $\mathbf{x}_{k}$ is the true target state at time $k, \hat{\mathbf{x}}_{k \mid k}$ is the estimate of the target at time $k$ given the measurements obtained up to that time, $\mathbf{P}_{k \mid k}$ is the error covariance for the estimate at time $k$ given all the measurements up to time $k$, and $\mathbf{J}_{k}$ is the FIM.

For a typical nonlinear system with Gaussian noise, the system dynamics are given by

$$
\begin{aligned}
\mathbf{x}_{k+1} & =\mathbf{f}\left(\mathbf{x}_{k}\right)+\mathbf{w}_{k} \\
\mathbf{z}_{k} & =\mathbf{h}\left(\mathbf{x}_{k}\right)+\mathbf{v}_{k}
\end{aligned}
$$

where $\mathbf{f}\left(\mathbf{x}_{k}\right)$ and $\mathbf{h}\left(\mathbf{x}_{k}\right)$ are the nonlinear process and measurement models respectively, and the noise variables $\mathbf{w}_{k}$ and $\mathbf{v}_{k}$ are uncorrelated, Gaussian, zero-mean white noises with covariances given by $\mathbf{Q}_{k}$ and $\mathbf{R}_{k}$ (i.e. $\mathbf{w}_{k} \sim \mathcal{N}\left(0, \mathbf{Q}_{k}\right)$ and $\mathbf{v}_{k} \sim \mathcal{N}\left(0, \mathbf{R}_{k}\right)$ ).

The recursive form of the Fisher Information Matrix for this system can be written s $^{\mathrm{a}}$

$$
\mathbf{J}_{k+1}=\left[\Phi_{k+1, k}^{T}\right]^{-1} \mathbf{J}_{k} \Phi_{k+1, k}^{-1}+\mathbf{H}_{k+1}^{T} \mathbf{R}_{k+1}^{-1} \mathbf{H}_{k+1}
$$

where $\Phi_{k+1, k}=\mathbf{F}_{k}=\nabla_{\mathbf{x}_{k}} \mathbf{f}\left(\mathbf{x}_{k}\right)$ and $\mathbf{H}_{k}=\nabla_{\mathbf{x}_{k}} \mathbf{h}\left(\mathbf{x}_{k}\right)$ are the Jacobians for the system dynamic model and the measurement model with respect to the estimation state. The recursion is initialized using $\mathbf{J}_{0}=\mathbf{P}_{0}^{-1}$.

\section{II.B. The Extended Kalman Filter}

The Kalman Filter has long been acknowledged as the most popular estimation algorithm. It is a recursive filtering method that is optimal and efficient ${ }^{\mathrm{b}}$ for linear, Gaussian, time-invariant dynamic systems, as well as being computationally efficient due to its recursive nature. To deal with systems that have nonlinear dynamics or nonlinear measurement models several modifications to the Kalman Filter have been proposed. A few examples are the Linearized Kalman Filter, the Extended Kalman Filter (EKF), and the Unscented Kalman Filter (UKF). This section presents the EKF for a system with nonlinear dynamics and a nonlinear measurement model, as described in Equation (4), and shows the close relationship between the EKF covariance and the Fisher Information Matrix, motivating the use of the FIM in trajectory design to enhance estimation performance.

The EKF algorithm is composed of a prediction step and an update step. The prediction step involves developing a state and covariance estimate of the next time step based on the current estimates and the system dynamics model. The update state involves processing the new measurement and updating the prediction made using the new information. The equations for the EKF are shown below.

Prediction phase:

$$
\begin{aligned}
\hat{\mathbf{x}}_{k+1 \mid k} & =\mathbf{f}\left(\hat{\mathbf{x}}_{k \mid k}\right) \\
\hat{\mathbf{z}}_{k+1 \mid k} & =\mathbf{h}\left(\hat{\mathbf{x}}_{k+1 \mid k}\right) \\
\mathbf{P}_{k+1 \mid k} & =\Phi_{k+1, k} \mathbf{P}_{k \mid k} \Phi_{k+1, k}^{T}+\mathbf{Q}_{k}
\end{aligned}
$$

Kalman Gain:

$$
\mathbf{K}_{k+1}=\mathbf{P}_{k+1 \mid k} \mathbf{H}_{k+1}^{T}\left[\mathbf{H}_{k+1} \mathbf{P}_{k+1 \mid k} \mathbf{H}_{k+1}^{T}+\mathbf{R}_{k+1}\right]^{-1}
$$

where $\Phi_{k+1, k}$ and $\mathbf{H}_{k}$ are, respectively, the Jacobians of the system dynamics and measurement model with respect to the state, evaluated at the predicted state $\hat{\mathbf{x}}_{k+1 \mid k}$.

Update phase:

$$
\begin{aligned}
\nu_{k+1} & =\mathbf{z}_{k+1}-\hat{\mathbf{z}}_{k+1 \mid k} \\
\hat{\mathbf{x}}_{k+1 \mid k+1} & =\hat{\mathbf{x}}_{k+1 \mid k}+\mathbf{K}_{k+1} \nu_{k+1} \\
\mathbf{P}_{k+1 \mid k+1} & =\mathbf{P}_{k+1 \mid k}-\mathbf{P}_{k+1 \mid k} \mathbf{H}_{k+1}^{T}\left[\mathbf{H}_{k+1} \mathbf{P}_{k+1 \mid k} \mathbf{H}_{k+1}^{T}+\mathbf{R}_{k+1}\right]^{-1} \mathbf{H}_{k+1} \mathbf{P}_{k+1 \mid k}
\end{aligned}
$$

Combining equations (8) and (12) and applying the Matrix Inversion Lemmac gives the inverse of the covariance propagation

$$
\mathbf{P}_{k+1 \mid k+1}^{-1}=\left[\Phi_{k+1, k} \mathbf{P}_{k \mid k} \Phi_{k+1, k}^{T}+\mathbf{Q}_{k}\right]^{-1}+\mathbf{H}_{k+1}^{T} \mathbf{R}_{k+1}^{-1} \mathbf{H}_{k+1}
$$

${ }^{\text {a }}$ The complete recursive derivation for the FIM is provided in Ristic et. al ${ }^{39}$

${ }^{\mathrm{b}}$ Achieves the Cramér-Rao Lower Bound

${ }^{\mathrm{c}}$ Matrix Inversion Lemma: $A-A B^{T}\left(B A B^{T}+C\right)^{-1} B A=\left(A^{-1}+B^{T} C^{-1} B\right)^{-1}$ 
For the case where there is no process noise $\left(\mathbf{Q}_{k}=\mathbf{0}\right)$, the inverse covariance propagation reduces to Equation (5) for the computation of the Fisher Information Matrix, ${ }^{39,42,43}$ showing that functionals based on the FIM make good objective functions for maneuver optimization since the FIM closely resembles the covariance of the EKF, which is widely accepted as a traditional measure of estimation performance.

\section{II.C. Vision-Based Target Localization Using EKF}

This section describes the problem of target localization using vision-only measurements obtained from a UAV flying above the target. As described in Section I.B.2, the images from the vision sensors can be converted into bearings-only measurements, which can be processed using an Extended Kalman Filter to determine the location of the target. The targets considered include a stationary target and a slow stochastic target that is free to move in the ground plane but for which no prior motion model is known. The UAV flight path is predefined and is assumed to be a circular trajectory orbiting the target at a constant altitude. The results presented show the estimation performance and the information obtained from the measurements using this predefined vehicle trajectory.

The details of the filtering algorithm are discussed next. The target dynamics model is assumed to be linear but the measurement model is still nonlinear, giving the following system dynamics,

$$
\begin{aligned}
\mathbf{x}_{k+1} & =\Phi_{k+1, k} \mathbf{x}_{k}+\mathbf{w}_{k} \\
\mathbf{z}_{k} & =\mathbf{h}\left(\mathbf{x}_{k}\right)+\mathbf{v}_{k}
\end{aligned}
$$

where $\Phi_{k+1, k}$ is the state transition matrix of the system from time $k$ to $k+1$ and $\mathbf{w}_{k}$ and $\mathbf{v}_{k}$ are the process and measurement noises, which are uncorrelated, Gaussian and white with zero mean and covariance $\mathbf{Q}_{k}$ and $\mathbf{R}_{k}$ respectively (i.e. $\mathbf{w}_{k} \sim \mathcal{N}\left(0, \mathbf{Q}_{k}\right)$ and $\mathbf{v}_{k} \sim \mathcal{N}\left(0, \mathbf{R}_{k}\right)$ ).

For the 3-D target localization scenario the target state to be estimated is $\mathbf{x}_{k}=\mathbf{t}_{k}=\left[\begin{array}{lll}t_{x} & t_{y} & t_{z}\end{array}\right]_{k}^{T}$. The measurement model involves two bearing measurements (described in Section I.B.2 and shown in Figure 4) and is given by

$$
\mathbf{h}\left(\mathbf{x}_{k}\right)=\left[\begin{array}{l}
\beta \\
\phi
\end{array}\right]=\left[\begin{array}{c}
\tan ^{-1}\left(\frac{p_{x}-t_{x}}{p_{y}-t_{y}}\right) \\
\tan ^{-1}\left(\frac{p_{z}-t_{z}}{\sqrt{\left(p_{x}-t_{x}\right)^{2}+\left(p_{y}-t_{y}\right)^{2}}}\right)
\end{array}\right]=\left[\begin{array}{c}
\tan ^{-1}\left(\frac{r_{x}}{r_{y}}\right) \\
\tan ^{-1}\left(\frac{r_{z}}{\sqrt{r_{x}^{2}+r_{y}^{2}}}\right)
\end{array}\right]
$$

where $\mathbf{p}_{k}=\left[\begin{array}{lll}p_{x} & p_{y} & p_{z}\end{array}\right]_{k}^{T}$ is the position of the vehicle, $\mathbf{t}_{k}=\left[\begin{array}{lll}t_{x} & t_{y} & t_{z}\end{array}\right]_{k}^{T}$ is the position of the target and $\mathbf{r}_{k}=\left[\begin{array}{lll}r_{x} & r_{y} & r_{z}\end{array}\right]_{k}^{T}$ is the relative vector between the vehicle and the target. The Jacobian of the measurement with respect to the target is

$$
\mathbf{H}_{k}=\left[\begin{array}{ccc}
-\frac{r_{y}}{r_{x}^{2}+r_{y}^{2}} & \frac{r_{x}}{r_{x}^{2}+r_{y}^{2}} & 0 \\
\frac{r_{x} r_{z}}{\left(r_{x}^{2}+r_{y}^{2}+r_{z}^{2}\right) \sqrt{r_{x}^{2}+r_{y}^{2}}} & \frac{r_{y} r_{z}}{\left(r_{x}^{2}+r_{y}^{2}+r_{z}^{2}\right) \sqrt{r_{x}^{2}+r_{y}^{2}}} & -\frac{\sqrt{r_{x}^{2}+r_{y}^{2}}}{\left(r_{x}^{2}+r_{y}^{2}+r_{z}^{2}\right)}
\end{array}\right]_{k}=\left[\begin{array}{l}
\mathbf{H}_{\beta} \\
\mathbf{H}_{\phi}
\end{array}\right]_{k}
$$

A simulation of the target localization problem, created in MATLAB, is used to analyze the target estimation performance for this baseline scenario. One hundred bearing measurements are taken by a vehicle following a circular trajectory around a target located at the origin. The position is measured in feet and the standard deviation of the sensor noise is assumed to be $\sigma=5 \mathrm{deg}$ which is fairly typical for commercially available bearing sensors. The EKF performance results for the different cases are summarized in Table 1 which provides: the end estimation error for each axis as well as the norm of the estimation error; the ending variance of the estimation error for each axis and the total sum of the variances; and the Fisher Information obtained about each axis as well as the total sum of information over all axes. An additional case involving a fast target is presented at the end of the section.

The case of a stationary target located at the origin is considered first. For this scenario, the process noise is zero since the target position is constant, so the filter parameters for the EKF are given by

$$
\Phi_{k, k-1}=\left[\begin{array}{ccc}
1 & 0 & 0 \\
0 & 1 & 0 \\
0 & 0 & 1
\end{array}\right], \quad \mathbf{Q}_{k}=\left[\begin{array}{ccc}
0 & 0 & 0 \\
0 & 0 & 0 \\
0 & 0 & 0
\end{array}\right], \quad \mathbf{R}_{k}=\left[\begin{array}{cc}
\sigma^{2} & 0 \\
0 & \sigma^{2}
\end{array}\right]
$$


The simulation is initialized using the values,

$$
\mathbf{x}_{0}=\left[\begin{array}{l}
0 \\
0 \\
0
\end{array}\right], \quad \hat{\mathbf{x}}_{0}=\left[\begin{array}{l}
20 \\
20 \\
20
\end{array}\right], \quad \mathbf{P}_{0}=\left[\begin{array}{ccc}
200 & 0 & 0 \\
0 & 200 & 0 \\
0 & 0 & 200
\end{array}\right]
$$

The initialization values shown above were selected arbitrarily. In practice, one can use a least squares approach with the first two measurements to develop an initial estimate of the target. Since the first two measurements are taken from locations very close to one another this will give a bad initial estimate, because the information provided by both measurements is similar, and therefore, the second measurement does not provide much new information. Another approach would be to use other available data. For example one could use the UAV altitude, one measurement, and an assumption that the target is on the ground to develop an initial target estimate. This approach will usually have better geometry than the case of initialization with two subsequent measurements and will yield a more accurate initial target estimate. A third approach is to use an a priori distribution model for the target if one is available.

The EKF results for the stationary target case are presented in Figure 6. The upper plots from left to right show: the ground track for the target localization results, the vehicle and target trajectories with the measurement line-of-sight vectors drawn in, and the information obtained in each axis as a function of the number of measurements. The lower plots from left to right show: the target estimation results for each axis, and the estimation error and standard deviation bounds for each axis. Figure 6 shows that the $Y$ axis estimation results improve faster than the $X$ axis due to the larger information provided initially by the trajectory in that direction. At 25 measurements the information about the $X$ and $Y$ dimensions is equal and the covariance or uncertainty in both dimensions also becomes the same. As the UAV circles around the target the $X$ and $Y$ information is increased in a sinusoidal manner due to the geometry of the vehicle trajectory. The $Z$ axis estimation performance remains the worst throughout as can be seen by looking at the standard deviation bounds and the information plot. This is because the UAV must remain above the target at all times resulting in poor geometry. The same trend can be seen in the problem of GPS navigation, where altitude is usually the most inaccurate value since the GPS satellites are always above the user.

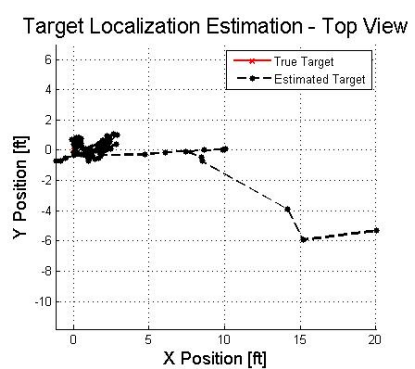

(a) Vehicle and Target Trajectories

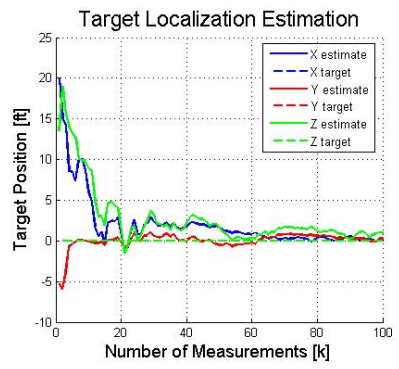

(c) Target Localization Estimation Results
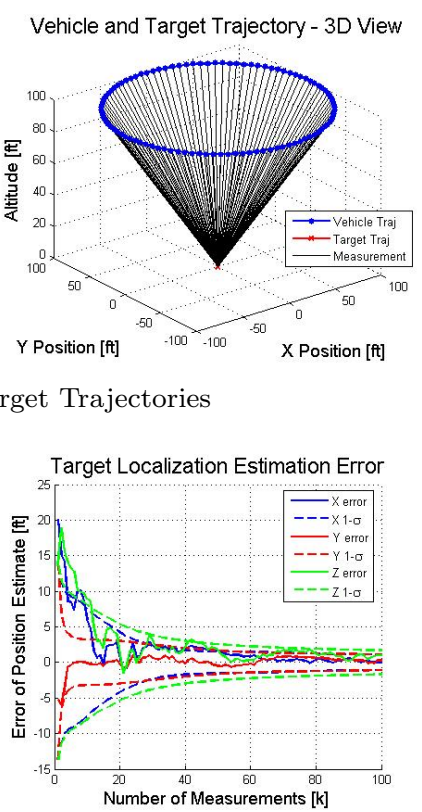

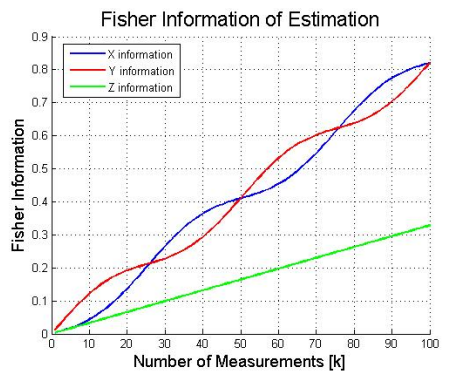

(b) Fisher Information

Figure 6: Localization of a stationary target using EKF algorithm in 3-D

The next case explored involves a target following a semi-circular trajectory. The target begins at the origin and moves around a semi-circular trajectory with a radius of $20 \mathrm{ft}$. The motion of the target, however, is unknown and no prior behavior models about the target are available. The target is therefore treated using 
a stochastic model which assumes a stationary process, but allows for unknown target motion by adjusting the assumptions on the process noise $\mathbf{Q}_{k}$. The filter parameters for this case are given by

$$
\Phi_{k, k-1}=\left[\begin{array}{lll}
1 & 0 & 0 \\
0 & 1 & 0 \\
0 & 0 & 1
\end{array}\right], \quad \mathbf{Q}_{k}=\left[\begin{array}{ccc}
7 & 0 & 0 \\
0 & 7 & 0 \\
0 & 0 & 0
\end{array}\right], \quad \mathbf{R}_{k}=\left[\begin{array}{cc}
\sigma^{2} & 0 \\
0 & \sigma^{2}
\end{array}\right]
$$

Here the nonzero process noise values for the $X$ and $Y$ variances represent the uncertainty in the motion of the target in these two directions. The $Z$ variance is still chosen to be zero since the target is assumed to remain on the ground. This is a reasonable assumption for ground targets traveling on fairly level terrain. If the terrain is more varied then some process noise may be added, but in general the motion along the $Z$ axis is expected to be considerably less than in the other directions. The actual values for the variances were selected as shown above for this specific case. In general the process noise selection represents a tradeoff, larger process noise values increase stability but decrease the accuracy of the estimation. The simulation is initialized as before, and the results for the curved target trajectory case are shown in Figure 7. The tracking performance is shown to be within $7 \mathrm{ft}$ of the actual target trajectory. For this scenario, the EKF diverges more often due to the nonlinearity of the target trajectory. It is interesting to note the close relationship between the Fisher Information and the covariance of the estimation. The information plot of Figure 7 shows that the Fisher Information about the $X$ and $Y$ coordinates increases in a sinusoidal pattern. This same sinusoidal pattern is seen in the 1- $\sigma$ bounds of the $X$ and $Y$ axes, showing that as information increases uncertainty decreases.

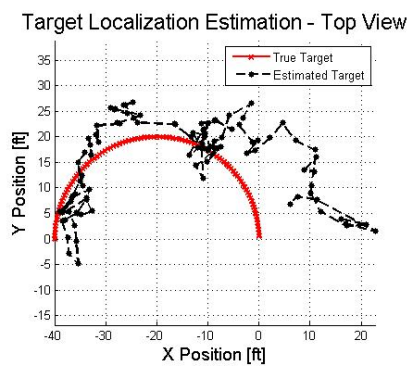

(a) Vehicle and Target Trajectories

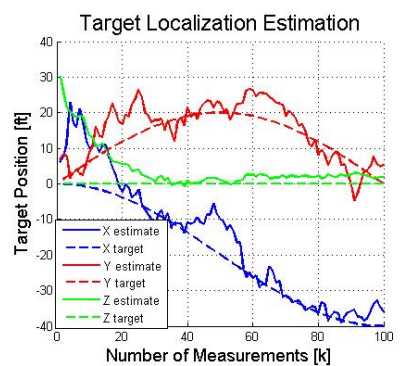

(c) Target Localization Estimation Results
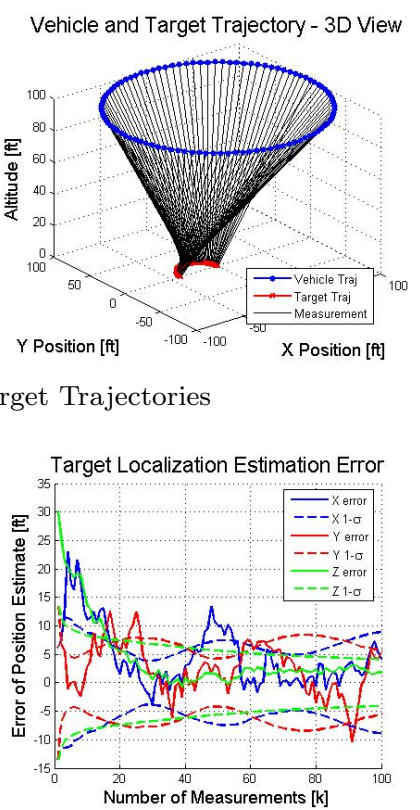

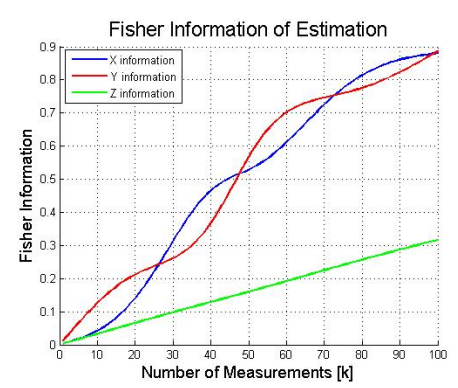

(b) Fisher Information

Figure 7: Localization of a turning target using EKF algorithm in 3-D

A final case considered involves tracking a fast moving target using the predefined UAV trajectory and the EKF estimation algorithm. For this scenario the target velocity is chosen to be $22 \mathrm{ft} / \mathrm{sec}$, which is half the cruise velocity of the UAV. The EKF parameters are given by,

$$
\Phi_{k, k-1}=\left[\begin{array}{lll}
1 & 0 & 0 \\
0 & 1 & 0 \\
0 & 0 & 1
\end{array}\right], \quad \mathbf{Q}_{k}=\left[\begin{array}{ccc}
1 & 0 & 0 \\
0 & 1 & 0 \\
0 & 0 & 0
\end{array}\right], \quad \mathbf{R}_{k}=\left[\begin{array}{cc}
\sigma^{2} & 0 \\
0 & \sigma^{2}
\end{array}\right]
$$

and the results for this case are presented in Figure 8. As seen in the plots, the EKF cannot track the target and the filter diverges. The motion of the target is too extreme, and even with several different values of 
Table 1: Summary of results for 3-D EKF Estimation

\begin{tabular}{||l|c|r|r|r||}
\hline Target Dynamics & & Estimation Error & Estimation Variance & Fisher Information \\
& $\mathrm{x}$ & 0.05 & 1.20 & 0.82 \\
& $\mathrm{y}$ & 0.31 & 1.18 & 0.82 \\
& $\mathrm{z}$ & 0.97 & 2.82 & 0.33 \\
& Total & 1.02 & 5.19 & 1.97 \\
\hline Semi-circular & $\mathrm{x}$ & 4.17 & 80.89 & 0.88 \\
& $\mathrm{y}$ & 5.26 & 30.66 & 0.89 \\
& $\mathrm{z}$ & 1.85 & 16.78 & 0.32 \\
& Total & 6.96 & 128.33 & 2.09 \\
\hline
\end{tabular}

process noise the filter cannot localize the target properly. The information plot of Figure 8 shows that the information content of the measurements provided by the ad-hoc circular trajectory is much lower than the previous cases, and that as the target moves farther away, the information tends to level off, indicating that the new measurements are not providing much new information.

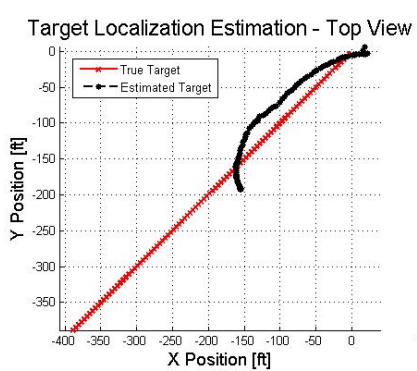

(a) Vehicle and Target Trajectories

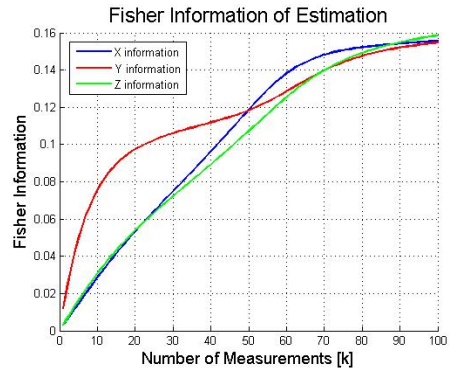

(b) Fisher Information
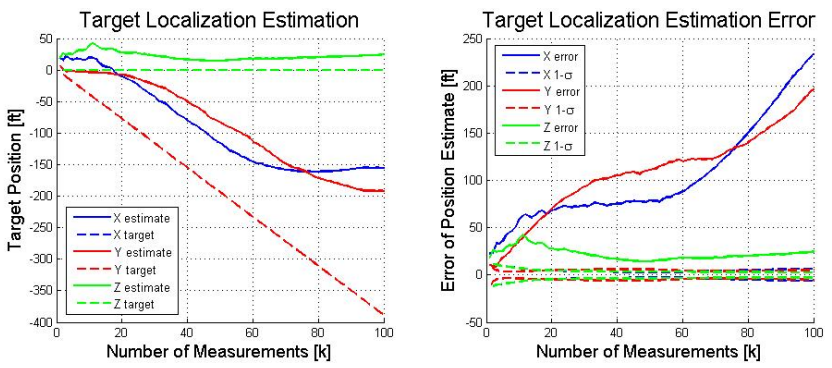

(c) Target Localization Estimation Results

Figure 8: Localization of a fast constant velocity target using EKF algorithm in 3-D

Using an EKF with a stochastic target model produces promising results for different types of target trajectories. Even though no initial model of target behavior is known, the EKF is able to track the target for the slow moving case. The approach taken in most past literature is to assume a model for the target behavior (typically stationary or moving with a constant velocity). If a correct model of target motion is incorporated the filter performance can be enhanced, however, the tradeoff is the obvious lack of flexibility. Since in practice it is rare to have a model of target behavior, especially for small UAVs used in areas in which the user has no prior information about the terrain or the targets it may encounter, it is of more value to have a filtering algorithm that is flexible enough to handle several types of target behaviors. Even though this approach works well for stationary and slow moving targets, the EKF with a stochastic target model cannot handle fast moving targets. Using an ad-hoc vehicle trajectory, the EKF algorithm is incapable of tracking fast targets and the filter diverges. The relationship between the information content of the set of 
measurements and the filter performance is shown in the cases above, motivating the development of vehicle trajectories that increase the amount of information provided by the measurements. Section III explains the process of developing such information-based UAV trajectories for 3-D bearings-only target localization and Section IV revisits the cases presented above using a simultaneous target estimation and vehicle trajectory optimization approach.

\section{Vehicle Trajectory Optimization}

The previous sections illustrate the dependence of the estimation performance on the vehicle trajectory. This is of considerable concern for the problem of target localization using vision based measurements, since the measurement model is highly nonlinear, and the estimation results become severely biased if the measurements do not provide much new information and proper estimation observability is not obtained. This section explores trajectory optimization for the 3-D bearings-only target localization problem, defining the Fisher Information Matrix and describing how to obtain an objective function based on the FIM. Several optimization scenarios are considered and the results are shown in the following sections.

It is important to note that the results in this section are obtained under the assumption that the true target location is known. Since the problem at hand is to estimate the location of the target, this is obviously a limiting assumption. The dependence of the optimization solution on the true target location has been one of the biggest problems associated with vehicle trajectory optimization, usually leading to a difficult nonlinear problem if the estimation and optimization are solved simultaneously. ${ }^{44}$ Nevertheless, exploring theoretical results for trajectory optimization using the true target location provides intuition about the expected performance of the trajectory optimization. Section IV revisits this issue and provides methods to combine the trajectory optimization and target estimation algorithms.

\section{III.A. Fisher Information Matrix for 3-D Bearings-Only Target Localization}

Since the Fisher Information Matrix captures the geometrical properties of the estimation problem, an information-theoretic framework provides a way of selecting which measurements bring the most additional or new information, thus enhancing the performance of the estimation. In addition to being related to the accuracy of the estimation, the FIM enjoys several useful properties which make it a good framework for the trajectory optimization problem. These properties include being symmetric and positive-definite (if estimation is observable), having a recursive form for computation, and being additive across multiple sensors and time-steps. The details for the computation of the FIM are provided below.

Recall from Section II.A that the recursive form of the Fisher Information Matrix is given by

$$
\mathbf{J}_{k}=\left[\Phi_{k, k-1}^{T}\right]^{-1} \mathbf{J}_{k-1} \Phi_{k, k-1}^{-1}+\mathbf{H}_{k}^{T} \mathbf{R}_{k}^{-1} \mathbf{H}_{k}
$$

For the 3-D bearings-only target localization problem the Jacobian of the measurement model with respect to the target was given by Equation (16) and is repeated below for convenience

$$
\mathbf{H}_{k}=\left[\begin{array}{ccc}
-\frac{r_{y}}{r_{x}^{2}+r_{y}^{2}} & \frac{r_{x}}{r_{x}^{2}+r_{y}^{2}} & 0 \\
\frac{r_{x} r_{z}}{\left(r_{x}^{2}+r_{y}^{2}+r_{z}^{2}\right) \sqrt{r_{x}^{2}+r_{y}^{2}}} & \frac{r_{y} r_{z}}{\left(r_{x}^{2}+r_{y}^{2}+r_{z}^{2}\right) \sqrt{r_{x}^{2}+r_{y}^{2}}} & -\frac{\sqrt{r_{x}^{2}+r_{y}^{2}}}{\left(r_{x}^{2}+r_{y}^{2}+r_{z}^{2}\right)}
\end{array}\right]_{k}=\left[\begin{array}{l}
\mathbf{H}_{\beta} \\
\mathbf{H}_{\phi}
\end{array}\right]_{k}
$$

Since the target is stationary or stochastic with a stationary process model the state transition matrix is the identity matrix. The noise is assumed to be uncorrelated and with constant covariance giving

$$
\Phi_{k+1, k}=\left[\begin{array}{ccc}
1 & 0 & 0 \\
0 & 1 & 0 \\
0 & 0 & 1
\end{array}\right], \quad \mathbf{R}_{k}=\left[\begin{array}{cc}
\sigma^{2} & 0 \\
0 & \sigma^{2}
\end{array}\right]
$$

Given these assumptions the FIM reduces to

$$
\mathbf{J}_{k}=\frac{1}{\sigma^{2}} \sum_{i=1}^{k} \mathbf{H}_{i}^{T} \mathbf{H}_{i}
$$


In the 3-D case $\mathbf{H}^{T} \mathbf{H}$ becomes

$$
\begin{aligned}
\mathbf{H}^{T} \mathbf{H} & =\left[\begin{array}{ccc}
\frac{r_{y}^{2}}{\left(r_{x}^{2}+r_{y}^{2}\right)^{2}} & -\frac{r_{x} r_{y}}{\left(r_{x}^{2}+r_{y}^{2}\right)^{2}} & 0 \\
-\frac{r_{x} r_{y}}{\left(r_{x}^{2}+r_{y}^{2}\right)^{2}} & \frac{r_{x}^{2}}{\left(r_{x}^{2}+r_{y}^{2}\right)^{2}} & 0 \\
0 & 0 & 0
\end{array}\right]+\left[\begin{array}{ccc}
\frac{r_{x}^{2} r_{z}^{2}}{\left(r_{x}^{2}+r_{y}^{2}+r_{z}^{2}\right)^{2}\left(r_{x}^{2}+r_{y}^{2}\right)} & \frac{r_{x} r_{y} r_{z}^{2}}{\left(r_{x}^{2}+r_{y}^{2}+r_{z}^{2}\right)^{2}\left(r_{x}^{2}+r_{y}^{2}\right)} & -\frac{r_{x} r_{z}}{\left(r_{x}^{2}+r_{y}^{2}+r_{z}^{2}\right)^{2}} \\
\frac{r_{x} r_{y} r_{z}^{2}}{\left(r_{x}^{2}+r_{y}^{2}+r_{z}^{2}\right)^{2}\left(r_{x}^{2}+r_{y}^{2}\right)} & \frac{r_{y}^{2} r_{z}^{2}}{\left(r_{x}^{2}+r_{y}^{2}+r_{z}^{2}\right)^{2}\left(r_{x}^{2}+r_{y}^{2}\right)} & -\frac{r_{y} r_{z}}{\left(r_{x}^{2}+r_{y}^{2}+r_{z}^{2}\right)^{2}} \\
-\frac{r_{x} z_{z}}{\left(r_{x}^{2}+r_{y}^{2}+r_{z}^{2}\right)^{2}} & -\frac{r_{y} r_{z}}{\left(r_{x}^{2}+r_{y}^{2}+r_{z}^{2}\right)^{2}} & \frac{\left(r_{x}^{2}+r_{y}^{2}\right)}{\left(r_{x}^{2}+r_{y}^{2}+r_{z}^{2}\right)^{2}}
\end{array}\right] \\
& =\mathbf{H}_{\beta}^{T} \mathbf{H}_{\beta}+\mathbf{H}_{\phi}^{T} \mathbf{H}_{\phi}
\end{aligned}
$$

The FIM for this 3-D case can also be written as

$$
\mathbf{J}_{k}=\frac{1}{\sigma^{2}} \sum_{i=1}^{k} \mathbf{H}_{i}^{T} \mathbf{H}_{i}=\frac{1}{\sigma^{2}} \sum_{i=1}^{k}\left(\mathbf{H}_{i_{\beta}}^{T} \mathbf{H}_{i_{\beta}}+\mathbf{H}_{i_{\phi}}^{T} \mathbf{H}_{i_{\phi}}\right)=\mathbf{J}_{k_{\beta}}+\mathbf{J}_{k_{\phi}}
$$

showing that the FIM is additive and that the FIM contributions of each sensor can be computed separately (assuming that the sensors are independent and uncorrelated). This is a very valuable property, as it allows for the inclusion of multiple sensors with different characteristics or sensors providing measurements at different times.

To obtain a better physical intuition of what the equations of the FIM shown above mean, it is advantageous to make the following substitutions. The magnitude of the vector for the 2-D top-view projection corresponding to the measurement of $\beta$ (see Figure 4) can be written as

$$
\left|\mathbf{r}_{a}\right|=\sqrt{r_{x}^{2}+r_{y}^{2}}
$$

The overall magnitude of the relative measurement vector is given by

$$
|\mathbf{r}|=\sqrt{r_{x}^{2}+r_{y}^{2}+r_{z}^{2}}
$$

The following trigonometric substitutions can be made

$$
\sin \beta=\frac{r_{x}}{\left|\mathbf{r}_{a}\right|}, \quad \cos \beta=\frac{r_{y}}{\left|\mathbf{r}_{a}\right|}, \quad \sin \phi=\frac{r_{z}}{|\mathbf{r}|}, \quad \cos \phi=\frac{\left|\mathbf{r}_{a}\right|}{|\mathbf{r}|}
$$

Using the above substitutions the FIM can be rewritten as

$$
\begin{aligned}
\mathbf{J}_{k}=\frac{1}{\sigma^{2}} \sum_{i=1}^{k}\left(\frac{1}{\left|\mathbf{r}_{i_{a}}\right|^{2}}\left[\begin{array}{ccc}
\cos ^{2} \beta_{i} & -\sin \beta_{i} \cos \beta_{i} & 0 \\
-\sin \beta_{i} \cos \beta_{i} & \sin ^{2} \beta_{i} & 0 \\
0 & 0 & 0
\end{array}\right]+\ldots\right. \\
\left.\qquad \frac{1}{\left|\mathbf{r}_{i}\right|^{2}}\left[\begin{array}{ccc}
\sin ^{2} \beta_{i} \sin ^{2} \phi_{i} & \sin \beta_{i} \cos \beta_{i} \sin ^{2} \phi_{i} & -\sin \beta_{i} \sin \phi_{i} \cos \phi_{i} \\
\sin \beta_{i} \cos \beta_{i} \sin ^{2} \phi_{i} & \cos ^{2} \beta_{i} \sin ^{2} \phi_{i} & -\cos \beta_{i} \sin \phi_{i} \cos \phi_{i} \\
-\sin \beta_{i} \sin \phi_{i} \cos \phi_{i} & -\cos \beta_{i} \sin \phi_{i} \cos \phi_{i} & \cos ^{2} \phi_{i}
\end{array}\right]\right)
\end{aligned}
$$

which shows that the range and bearing information are separable in the FIM. It is convenient to rewrite the FIM using these trigonometric substitutions, especially when computing norms of the FIM, to preserve this separation between range and angular information. This form of the matrix suggests that maximization of the FIM involves lowering the range to the target and increasing the angular separation between measurements, which supports the intuition developed in Section I.B.3.

\section{III.B. Selection of an Objective Function Based on Matrix Measures}

Since maximizing the FIM involves maximizing a matrix, it is desirable to find a real-valued scalar function based on the FIM to serve as an objective function in the trajectory optimization problem. Going from a matrix to a scalar function results in a loss or compression of information, leading to a weaker optimization metric. Careful analysis of different possible functions is necessary to ensure that the information of interest to the estimation problem is properly captured. Furthermore, it is desirable to choose a function that is well structured for optimization (contains the fewest local extrema, is equally sensitive to all optimization 
variables, etc). As mentioned before, the FIM is symmetric and at least positive semi-definite, therefore its eigenvalues are real and positive (or zero). The eigenvalues of the FIM are related to the uncertainty ellipsoid of the target estimation, since the FIM is related to the estimation error covariance. More specifically, the length of each axis of the uncertainty ellipsoid is given by one over a squared eigenvalue, for each eigenvalue of the FIM. Therefore, maximizing the eigenvalues of the FIM leads to a smaller uncertainty ellipsoid and a more accurate estimation.

A few of the most common matrix measures are described below. The D-optimality criterion is based on the determinant of the FIM and is given by

$$
f(\mathbf{J})=-\log \operatorname{det}\{\mathbf{J}\}
$$

As the determinant of a symmetric positive definite matrix is given by the multiplication of its eigenvalues, the D-optimality criterion results in the minimization of the volume of the uncertainty ellipsoid. The determinant is a very popular choice of matrix measure and is used widely in the literature. One advantage of the Doptimality criterion is that it is invariant to parameter scale changes and linear transformations. ${ }^{44}$ The next function considered is the E-optimality condition, which uses the maximum eigenvalue of the inverse of the FIM and is given by

$$
f(\mathbf{J})=\max _{i}\left\{\operatorname{eig}\left(\mathbf{J}^{-1}\right)\right\}
$$

Optimizing using the E-optimality criterion leads to the minimization of the length of the largest axis of the uncertainty ellipsoid. A third function is the A-optimality criterion, which involves computing the trace of the inverse of the FIM. Its equation is given by

$$
f(\mathbf{J})=\operatorname{Tr}\left\{\mathbf{J}^{-1}\right\}
$$

and it is designed to minimize the average variance of the estimates. The main drawback of using the functions shown above is that they are not monotonic functions, resulting in local minima and maxima in the objective function. This makes the optimization quite sensitive to initial conditions and may cause the optimizer to get stuck in a local minimum if not initialized properly. The last function considered is the sensitivity criterion given by

$$
f(\mathbf{J})=-\operatorname{Tr}\{\mathbf{J}\}
$$

This criterion involves maximizing the trace of the FIM, which for a symmetric positive definite matrix is the sum of the eigenvalues. The sensitivity criterion is the easiest to compute and provides a monotonic optimization function; yet, it is not stable and could result in a singular FIM. It is typically used only to initialize the optimization problem. These are the most widely used matrix measures. Uciński provides a complete discussion on possible matrix measures for the FIM. ${ }^{44}$

A sensor placement exercise served as a benchmark for testing and comparing these functions based on the FIM. The algorithm uses a gradient descent method to find a set of $N$ optimal UAV measurement locations in 3 -D space with a fixed altitude constraint. This sensor placement case is used to test the performance of the candidate objective functions to determine which one is best suited to the problem. The selected objective function must capture the dependence of the estimation on the angular separation between measurement locations and the relative range to the target (as described above and in Section I.B.3). The first function, based on the determinant of the FIM, is very sensitive to changes in range but exhibits far less sensitivity to changes in angular separation. The optimization tends to reduce the range as much as possible, resulting in a sensor configuration that places all the points directly above the target (since, for a fixed altitude, this is the position of lowest relative range). This result is obviously undesirable since it leads to an unobservable estimation for the bearings-only estimation problem. Another test performed on the determinant function, but holding the range fixed, shows that the optimization algorithm separates the points equally. This confirms that the determinant function does contain information about the angular dependence between the measurements, but due to the high sensitivity of the determinant function to the relative range, this angular dependence is essentially ignored. It is important to mention that the determinant function is not dismissed lightly. In general, the D-optimality criterion captures most of the essential dependencies of the estimation on the measurement locations and is widely used in the literature as the optimization function of choice. Most of the literature uses the D-optimality criterion in a two-dimensional setting, however, in the 3-D case 
the sensitivity to range overshadows the sensitivity to angular separation between measurements, making the optimization quite difficult.

Next the sensitivity criterion involving the trace of the FIM is explored as an optimization function. The trace function results in a configuration which completely ignores the angular separation between measurements and optimizes only the range. Again, as in the case of the determinant function, the sensor placement optimization places all the measurement points directly overhead the target, resulting in an unobservable estimation. Furthermore, analytically deriving the trace of the FIM and simplifying the expression gives

$$
\operatorname{Tr}\{\mathbf{J}\}=\mathbf{J}_{k}=\frac{1}{\sigma^{2}} \sum_{i=1}^{k}\left(\frac{1}{\left|\mathbf{r}_{i_{a}}\right|^{2}}+\frac{1}{\left|\mathbf{r}_{i}\right|^{2}}\right)
$$

showing that the information about the dependence of the estimation on the angular separation between measurements is completely lost.

The remaining functions, the E-optimality and the A-optimality criteria, result in very similar measurement configurations. They both return sets of measurement locations that are evenly spaced in a circular pattern above the target. This circular sensor configuration shows the tradeoff between reducing the range to the target, while still maintaining as much orthogonality between measurements as possible. Furthermore, both the A-optimality and E-optimality criteria exhibit faster convergence and higher stability in the optimization and are less sensitive to local minimums. Since the A-optimality criterion involves all the eigenvalues of the inverse of the FIM, it represents the uncertainty in all dimensions equally, and is therefore chosen over the E-optimality criterion as the best optimization function. It is interesting to note that the expression for the A-optimality criterion closely resembles the expression for position dilution of precision (PDOP), which is commonly exploited in GPS navigation to select the configuration of satellites that gives the most accurate position estimate, showing that the A-optimality criterion captures the geometric system dependencies. Furthermore, the A-optimality criterion is the most physically significant function for the estimation problem since it is based on minimizing the individual variances of the estimates. The following sections show the results for the optimization of UAV measurement locations for several different scenarios using the A-optimality criterion.

\section{III.C. Trajectory Optimization Results}

Two main scenarios are considered for the problem of optimizing the trajectory of a UAV performing target localization. The first case examines the problem of finding the best sensing locations for a fixed number of measurements. This scenario is applicable to a UAV with a limit on the amount of measurements it can take (due to bandwidth or storage constraints, for example). It can also be used for the case of placing multiple UAVs which can collaborate to provide a vehicle estimate. The second scenario examines the problem of trajectory optimization for a single UAV with motion constraints. The rate at which measurements are taken is fixed and the motion constraints limit the amount the vehicle can travel and turn between measurements. The optimization for both scenarios is implemented using a gradient descent numerical method with the objective function given by the A-optimality criterion. The gradient descent algorithm uses a polar coordinate approach, varying the range to the target and the angular separation between the measurement locations. The results are provided in the following sections.

\section{III.C.1. Optimization of Measurement Locations Using Sensor Placement Techniques}

This section describes the case of trajectory optimization for a UAV flying at a fixed altitude above a stationary target taking a fixed number of measurements $(N)$ of the target. The optimization uses a gradient descent method which numerically computes the derivative with respect to the range and bearing for each measurement location and updates the positions by taking steps proportional to the computed derivative. The optimal sensor placement configuration is given by the measurement locations evenly spaced around a circle directly above the target. This is not a surprising result since a circular trajectory is widely accepted in literature as the optimal trajectory and supports the geometric intuition provided in Section I.B.3. The UAV trajectory is obtained by joining the measurement locations and the results are shown in Figure 9 for the case of $N=15$.

The optimization was performed for several different values of $N$. As the number of measurements increases the trajectory approaches a circle at a fixed altitude centered on the target. An interesting result 


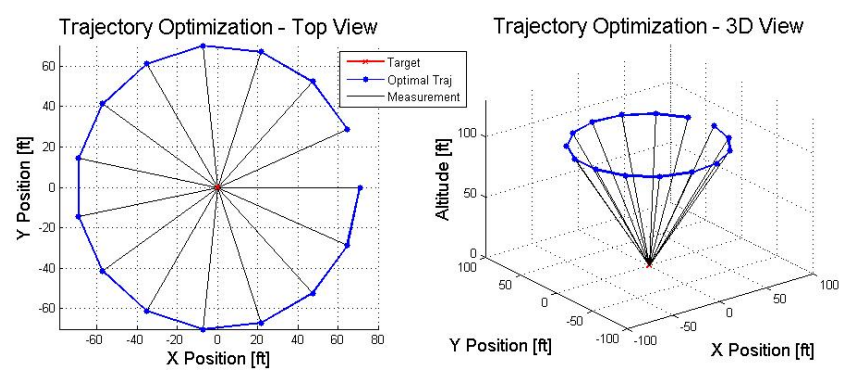

Figure 9: Optimization of vehicle trajectory using sensor placement techniques

is that the ratio of the radius of the circular trajectory to the altitude above the target is always close to $0.7( \pm 0.02)$ regardless of the number of measurements. This demonstrates the tradeoff between minimizing the relative range to the target and maintaining proper angular separation between measurements. If only the range is considered, all the measurements would be taken directly above the target, as this is the closest point within the altitude constraint, leading to a radius to altitude ratio of zero. If the angular separation is the only consideration the measurement vectors would be chosen to be orthogonal and the ratio between the radius and the altitude would be 1 . Since the optimal radius is 0.7 , this shows that there is a compromise between proximity to the target and angular separation of the measurements. These results scale with altitude, and the 0.7 ratio remains the same for several different altitudes.

The task of simultaneously optimizing $N$ measurement locations has several issues. The first is that the optimization is a multimodal problem of high dimensionality. The objective function is dependent upon all $N$ measurements and for the general 3-D case the gradient vector has $3 N$ terms $(2 N$ with a fixed altitude constraint). As $N$ increases, the computational load required to perform the optimization increases as well. Another complication is that as $N$ increases, the dependencies of the objective function on the measurement locations become more complex, making the optimization quite difficult. For the bearings-only case, the optimal configuration is not unique, as the spacing between the measurements is important and not the actual bearing locations, leading to infinitely many optimal configurations. This can be remedied by fixing the bearing information for one of the measurement locations and optimizing over the rest. Furthermore, as $N$ increases the objective function has a growing number of local extrema causing severe sensitivities to initialization and often leading to suboptimal solutions if not initialized properly. This is the most difficult issue to address, since unless there is some prior insight about the problem, it is often unclear what a proper choice of initial measurement configuration should be. Another issue with improper initialization of the optimization is that the algorithm tends to cluster the measurement locations. An example of this suboptimal configuration is shown in Figure 10, where the five-point trajectory optimization results in clusters of two points on each end of a line and one point in the center. The cyan lines show the progress of the optimization and the straight blue line shows the resulting "optimal" trajectory. This suboptimal solution occurs because of the assumption that the noise between the different measurements is spatially uncorrelated. ${ }^{44}$ This result is not practical and can be avoided by initializing the optimization close to the global minimum.

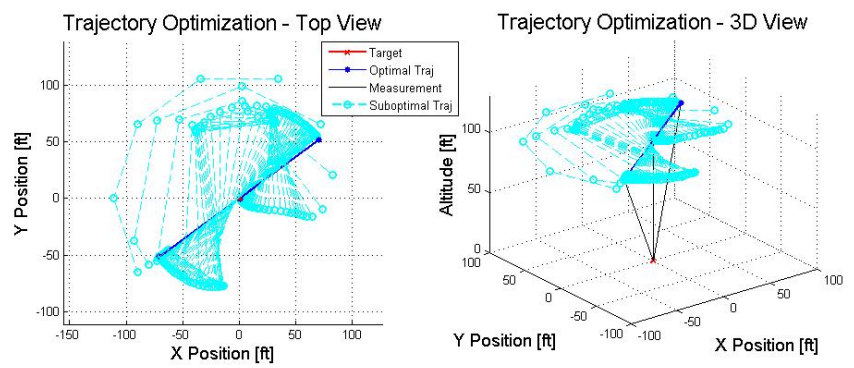

Figure 10: Sensor clustering due to improper initialization

The intuition about the problem and the insight provided by the sensor placement case with low values of $N$ suggest that the optimal solution tends to a circle of optimal radius above the target, with the measurement locations evenly distributed around it. Overall, the sensor placement case is a useful benchmark for providing 
intuition about the problem and for determining which objective function is best suited to the trajectory optimization task. The next section explores the inclusion of vehicle motion constraints to design trajectories for a single UAV that are dynamically feasible.

\section{III.C.2. Trajectory Optimization With Vehicle Motion Constraints}

In this section, the vehicle is constrained to move with a constant velocity and to have a maximum allowable turn rate. The optimization is initialized with a starting vehicle position and orientation, and uses the Aoptimality criterion to determine the subsequent trajectory points that maximize the amount of information while remaining within the vehicle's velocity and turn rate constraints. A single-point optimization strategy is used, and the Fisher Information Matrix is updated recursively at every time step. Several cases of trajectory optimization are considered. The first case assumes a stationary target located at the origin and a UAV initial position of $\mathbf{p}_{0}=\left[\begin{array}{lll}100 & 0 & 100\end{array}\right]^{T}$ with an initial heading of $\frac{\pi}{2}$ (North). These are the same initial conditions assumed in Section 2, where the UAV was made to fly a circular trajectory around the target. In this case, the trajectory is computed by the optimizer for 50 measurements and the results are presented in Figure 11. Of interest is that after the initial spiral, the UAV follows a circular orbit with a radius of approximately $70 \mathrm{ft}$. Since the altitude is $100 \mathrm{ft}$, this case illustrates the results from the previous section where the optimal radius to altitude ratio is found to be 0.7. Another interesting observation is that the information provided by this optimal trajectory in the $X$ and $Y$ axes is almost equivalent to the information provided by the ad-hoc circular UAV trajectory used in Section 2 (see Figure 6), however the number of measurements taken for the optimal trajectory is 50, whereas the ad-hoc trajectory required 100 measurements to reach this amount of information. This shows that by using a trajectory optimization algorithm and placing the measurement locations efficiently, the number of measurements required to achieve a certain information threshold can be drastically reduced. Unfortunately, due to the poor geometry with respect to the $Z$ axis, the information about this dimension cannot be changed much.

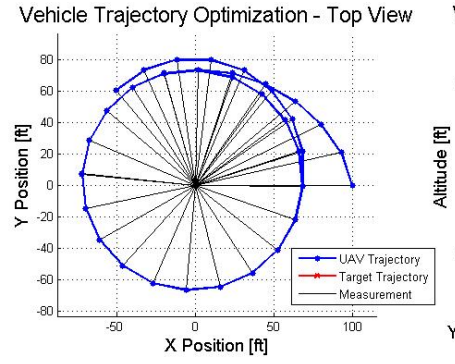

(a) Vehicle and Target Trajectories

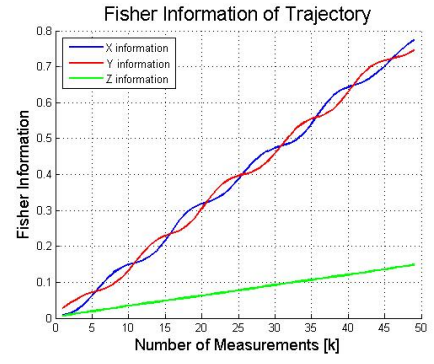

(b) Fisher Information

Figure 11: Trajectory optimization with motion constraints, stationary target, case 1

The next case considered places the UAV at an initial position which is farther from the target $\left(\mathbf{p}_{0}=\right.$ $\left[\begin{array}{lll}200 & 0 & 100\end{array}\right]^{T}$ ). The resulting trajectory is shown in Figure 12. Here the UAV begins by spiralling toward the target, but instead of converging to a circular orbit it attempts to reduce the relative range to the target as much as possible. This behavior increases the amount of information provided in the $X$ and $Y$ dimensions as shown in the lower right plot of Figure 12. The sharp increase in information around measurements 15 and 35 correspond to the points on the trajectory that are nearest to the target, showing the sensitivity of the Fisher Information to the relative range.

The next few cases study the effect of the UAV turn rate constraint. The trajectory optimization is performed for a 100 measurement trajectory and the maximum UAV turn rate is varied. The first scenario uses a maximum allowable turn rate of $10 \mathrm{deg} / \mathrm{sec}$. The UAV spirals towards the target, reducing the relative range to the target while maintaining a heading which is nearly orthogonal to the line-of-sight vector to the target. The UAV trajectory converges on to an orbit around the target, with the target off-center. Additionally, the information rate is higher and the increase in information is sharper when the vehicle passes closest to the target. Therefore the optimal information-theoretic move is to minimize the relative range to the target as much as possible within the vehicle turn rate constraint.

When the turn rate is increased to $15 \mathrm{deg} / \mathrm{sec}$, the resulting trajectory is provided in Figure 14 and is 


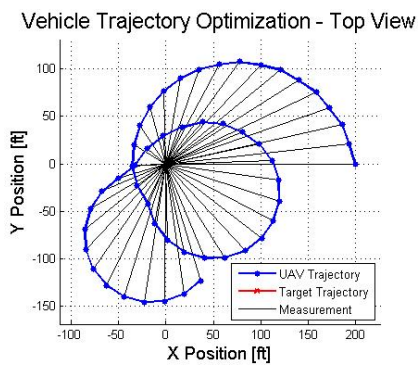

(a) Vehicle and Target Trajectories

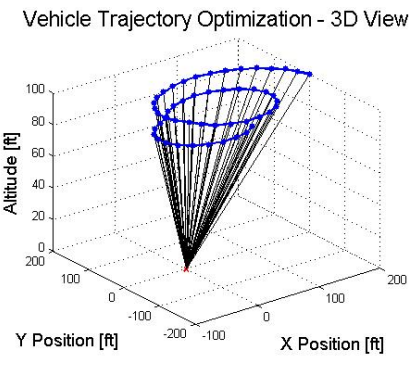

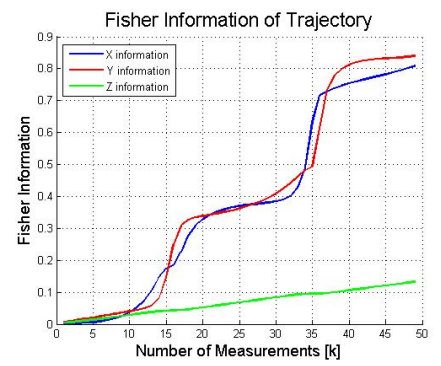

(b) Fisher Information

Figure 12: Trajectory optimization with motion constraints, stationary target, case 2

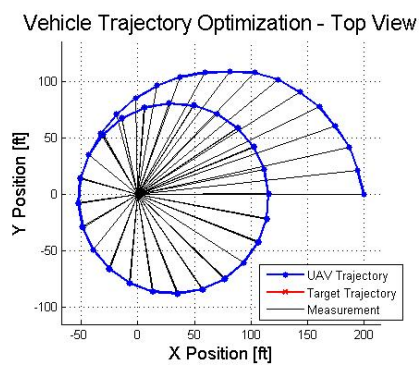

(a) Vehicle and Target Trajectories

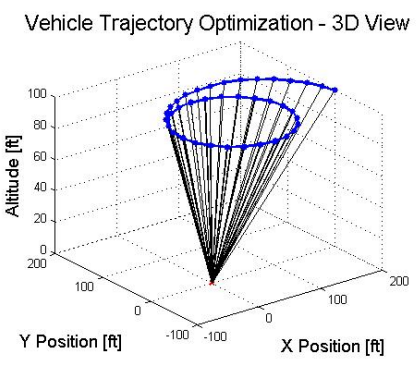

Figure 13: Trajectory optimization with turn constraint of $10 \mathrm{deg} / \mathrm{sec}$

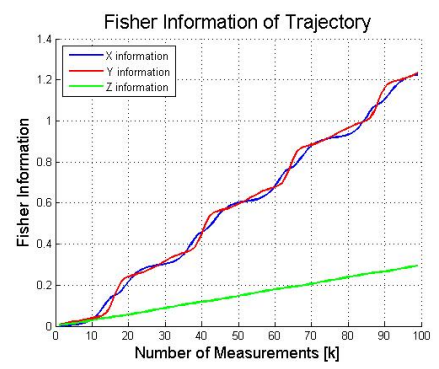

(b) Fisher Information

shown to spiral close to the target twice before converging onto a circle around the target. This time, the circle is centered on the target. Again the two sharp increases in information correspond to when the UAV is nearest to the target. The final case considered is for a maximum turn rate of $20 \mathrm{deg} / \mathrm{sec}$. The results for

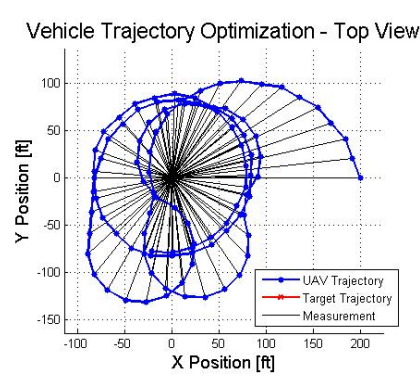

(a) Vehicle and Target Trajectories

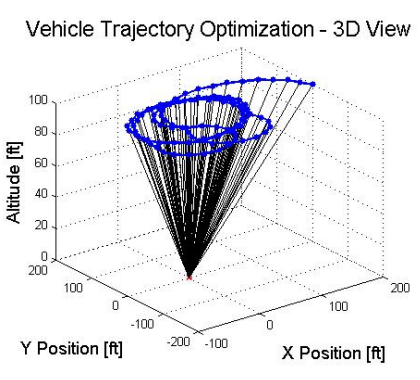

Figure 14: Trajectory optimization with turn constraint of $15 \mathrm{deg} / \mathrm{sec}$

this scenario are shown in Figure 15. The UAV once again spirals into the target, this time passing almost directly overhead the target. The information is drastically increased during this first pass. The UAV then circles around and converges to a limit cycle centered on the target, where the information increases at a constant rate. Note that the total amount of information increases as the constraints are relaxed. For the cases considered, $10 \mathrm{deg} / \mathrm{sec}, 15 \mathrm{deg} / \mathrm{sec}$ and $20 \mathrm{deg} / \mathrm{sec}$, the average total information for the $X$ and $Y$ axes was $1.22,1.58$, and 2.30 , respectively. This shows that the more restricted the vehicle is, the less capable it is of obtaining the best possible trajectory. The maximum turn rate study shows that different UAV constraints cause different orbiting behavior, however, the information-theoretic framework produces trajectories that have the basic properties of reducing the relative range to the target as much as possible, within the vehicle constraints, and maintaining a heading that is nearly orthogonal to the line-of-sight to the target.

Trajectory optimization for moving targets is considered next. The first scenario involves a target fol- 


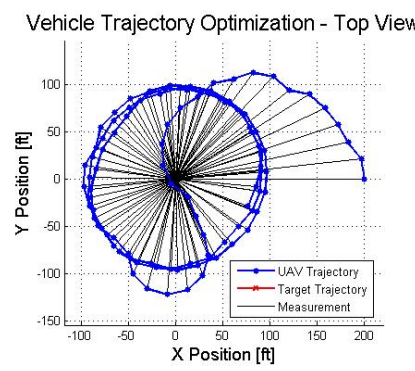

(a) Vehicle and Target Trajectories

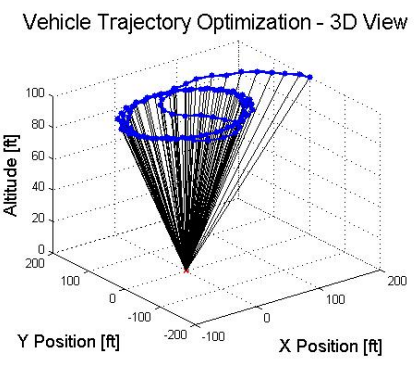

Figure 15: Trajectory optimization with turn constraint of $20 \mathrm{deg} / \mathrm{sec}$

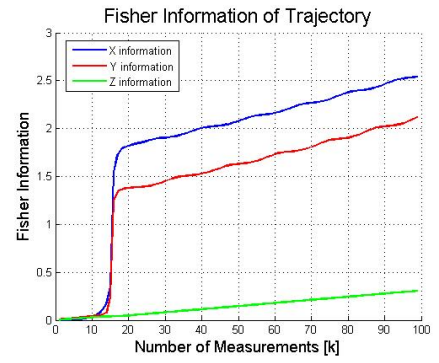

(b) Fisher Information

lowing a semi-circular trajectory with a $20 \mathrm{ft}$ radius. The UAV is restricted to maintain a cruise speed of 44 $\mathrm{ft} / \mathrm{sec}$ and has a maximum desired turn radius of $12 \mathrm{deg} / \mathrm{sec}$. The starting position is $\mathbf{p}_{0}=\left[\begin{array}{lll}200 & 0 & 100\end{array}\right]^{T}$ and the initial heading is $\frac{\pi}{2}$. The results are shown in Figure 16. Again the basic spiralling behavior is present, maintaining an orthogonal motion to the line-of-sight vector to the target while reducing relative range. As the UAV passes near the target the information increases more rapidly, as before.

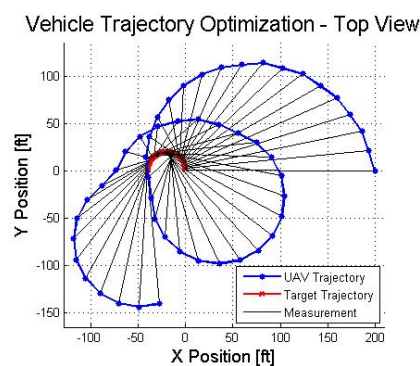

(a) Vehicle and Target Trajectories

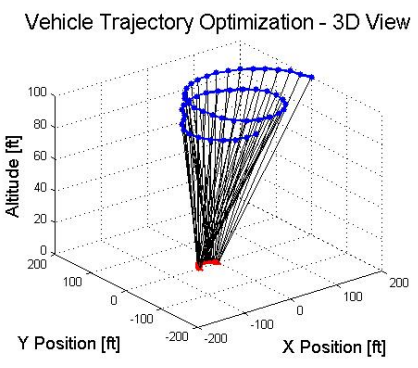

Position [t]

Figure 16: Trajectory optimization for slow moving target

A faster moving targets case is considered as well. The target moves at a constant velocity of $22 \mathrm{ft} / \mathrm{sec}$, which is half of the UAV velocity. The results for this case are shown in Figure 17. The UAV spirals towards the target but cannot close in because of the fast target motion. It then moves alongside the target until it has passed it and finally spirals around to the other side. Note the sharp information increase obtained when the UAV crosses over the target. This is due to the reduction in uncertainty provided by the geometry of the measurements.

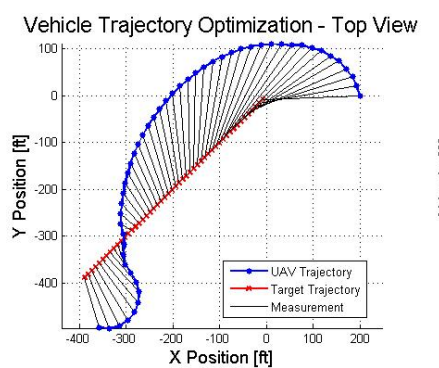

(a) Vehicle and Target Trajectories

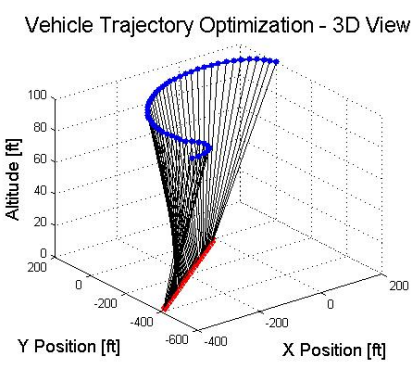

X Position [t]

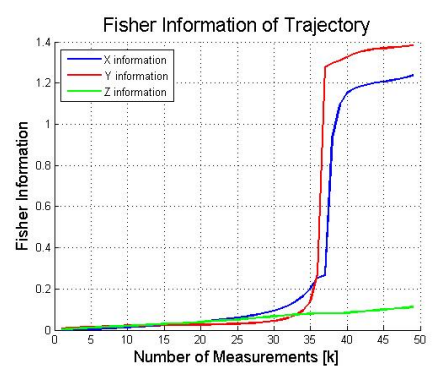

(b) Fisher Information

Figure 17: Trajectory optimization for fast constant velocity target

One limitation of using the Fisher Information Matrix in the moving target scenarios is that the FIM is computed using the position of the target at the particular time the corresponding measurement is taken. The total information reflects the summation over all the measurements. However, since the target is moving 
and its position is changing, the information provided by the old measurements is not as useful as that of the new ones. The FIM framework accounts for this by using a proper transition matrix that represents the motion of the target. However, in our case, the behavior model of the target is unknown and therefore the transition matrix is assumed to be an identity matrix. The result of this inaccuracy is that the information provided by older measurements is not as valuable as that of the newer information and, therefore, the total information used in the problem misrepresents the reality of the situation.

The UAV trajectories presented in this section show that, even though the circular trajectory above the target is widely accepted as the optimal trajectory, if not at the optimal radius, for a UAV with motion constraints, the optimal information-theoretic action is to spiral towards the target, minimizing the relative range and increasing the angular separation between measurements. This spiral behavior maximizes the information by reducing the relative range as much as possible instead of circling above the target where errors in the $X$ and $Y$ axes are improved but at a slower rate. The approach shown in this section provides a better way to design UAV trajectories, which is based on minimizing the estimation error and maximizing the information provided by each measurement, and which is modular enough to handle stationary and moving targets.

\section{Simultaneous Trajectory Optimization and Target Estimation}

The main issue with information based optimization strategies is the dependence of the objective function on the parameters to be estimated. As shown in Section III, the Fisher Information Matrix is a function of the parameters to be estimated, which in this case is the target location. This issue is prevalent throughout the literature and is best described by Cochran ${ }^{8}$ as, "You tell me the value of $\theta$, and I promise to design the best experiment for estimating $\theta$." Since in practice the trajectory planner has no access to the true target state, the best that can be done is to use an a priori distribution on the target location ${ }^{29}$ and to perform the target estimation and vehicle trajectory optimization simultaneously. There are obvious limitations to this approach and, since combining the estimation and optimization usually leads to a highly nonlinear problem, convergence cannot be guaranteed. However, for the problem of 3-D bearings-only target tracking, if the estimation is initialized properly and the optimization is updated at a fast enough rate, the simultaneous estimation and optimization approach provides the desired performance.

For this simultaneous estimation and optimization problem, the trajectory optimization is performed by using the most current value of the target estimation to form the FIM, and then minimizing the trace of the inverse of the FIM (using the A-optimality criterion described in Section III). Measurements are taken every 0.5 seconds and the estimation and optimization routines are run in sequence, updating both results at every time step. The same cases of target motion as described in Section II are analyzed and the results are summarized in Table 2.

The stationary target scenario is considered first. The filter initialization and UAV constraints are the same as in Section II. The UAV initial position is given by $\mathbf{p}_{0}=\left[\begin{array}{lll}200 & 0 & 100\end{array}\right]^{T}$, which is much farther than the initial position given in Section II of $\mathbf{p}_{0}=\left[\begin{array}{lll}100 & 0 & 100\end{array}\right]^{T}$. The results for the simultaneous trajectory optimization and target estimation for the stationary target case are provided in Figure 18. The UAV trajectory follows the spiral shape described in Section III. The convergence of the estimation is much faster, and the resulting mean and variance for the $X$ and $Y$ dimensions is more accurate than the case presented in Section II (see Table 2), and using only half the measurements. Furthermore, the $X$ and $Y$ information are also shown to be higher than in the case in Section II. The estimation performance for the $Z$ axis is worse however, since only half the measurements are used, and the trajectory optimization cannot improve the information obtained about the $Z$ axis well. It is interesting to note that the information in the $X$ and $Y$ axes increases sharply when the UAV crosses over the target the second time, and, correspondingly, the estimation performance drastically improves. Overall, even though the UAV is farther away from the target than in the previous case, and less measurements are taken, the estimation performance is better, as the optimized trajectory provides higher information, reducing the uncertainty in the estimation.

The next scenario considers a target following a semi-circular trajectory. As before, no prior target motion models are available and a stochastic model of the target is used in the EKF. The results for the turning target case are provided in Figure 19. The UAV trajectory again resembles the results shown in Section III. A sharp information gain can be seen as the UAV comes close to the target, around 15 measurements, causing a corresponding decrease in uncertainty and increase in estimation accuracy. The 

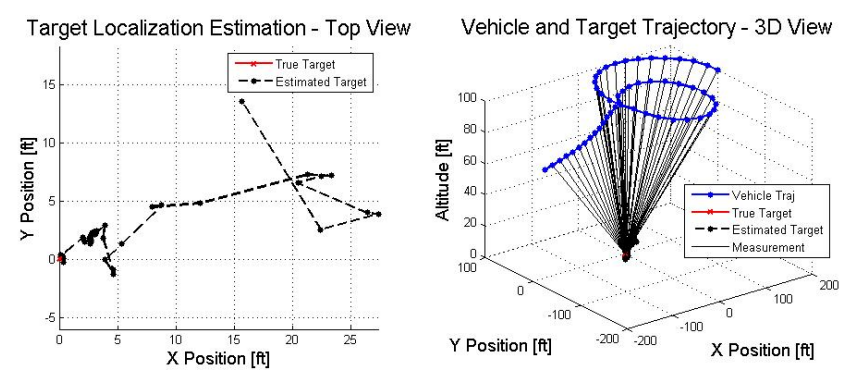

(a) Vehicle and Target Trajectories
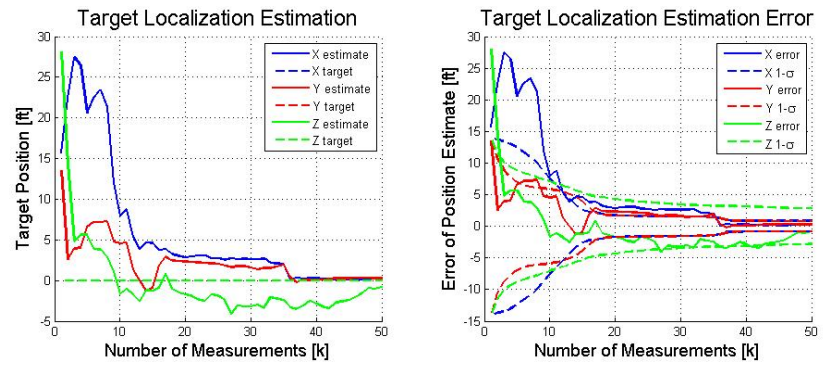

(c) Target Localization Estimation Results

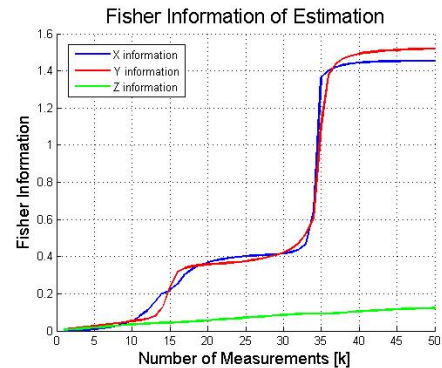

(b) Fisher Information

Figure 18: UAV trajectory optimization and EKF estimation results for stationary target localization

overall estimation performance is better than in Section II, showing a lower error, much lower variance, and increased information with only half the measurements (see Table 2).

Table 2: Summary of results for 3-D EKF Estimation with Trajectory Optimization

\begin{tabular}{||l|c|r|r|r||}
\hline Target Dynamics & & Estimation Error & Estimation Variance & Fisher Information \\
\hline Stationary & $\mathrm{x}$ & 0.23 & 0.71 & 1.45 \\
& $\mathrm{y}$ & 0.28 & 0.68 & 1.52 \\
& $\mathrm{z}$ & -0.81 & 7.92 & 0.12 \\
& Total & 0.89 & 9.30 & 3.09 \\
\hline \multirow{5}{*}{ Semi-circular } & $\mathrm{x}$ & -2.21 & 4.35 & 1.15 \\
& $\mathrm{y}$ & 2.05 & 11.42 & 1.08 \\
& $\mathrm{z}$ & 0.86 & 9.86 & 0.15 \\
& Total & 3.14 & 25.62 & 2.38 \\
\hline
\end{tabular}

The final fast moving target case is considered as well. The results for the simultaneous estimation and optimization are shown in Figure 20. Although the errors are quite large, the EKF manages to track the target and the filter remains stable, unlike the case presented in Section II using the ad-hoc circular trajectory. Furthermore, the information provided by the measurements using the optimized UAV trajectory is much higher than in Section II using the circular trajectory. This case clearly illustrates the benefits of optimizing and re-planning the UAV trajectory in real-time using an information-based approach.

The simultaneous trajectory optimization and target estimation shows that enhanced estimation performance can be obtained by optimizing the target trajectory using an information metric in real-time. For the cases described above, the estimation converges rapidly, the EKF algorithm is considerably more stable than before, and the estimation achieves accurate results within only 50 measurements. The total amount of information obtained using the optimized trajectory is shown to be similar to the information gained over the ad-hoc trajectory presented in Section II, but with only half the measurements. The processing time required to perform the computations for these two algorithms is usually well within the allotted time of 


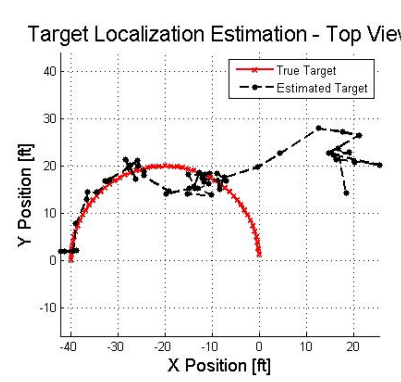

(a) Vehicle and Target Trajectories

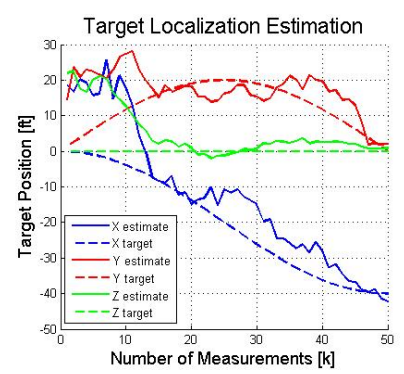

(c) Target Localization Estimation Results
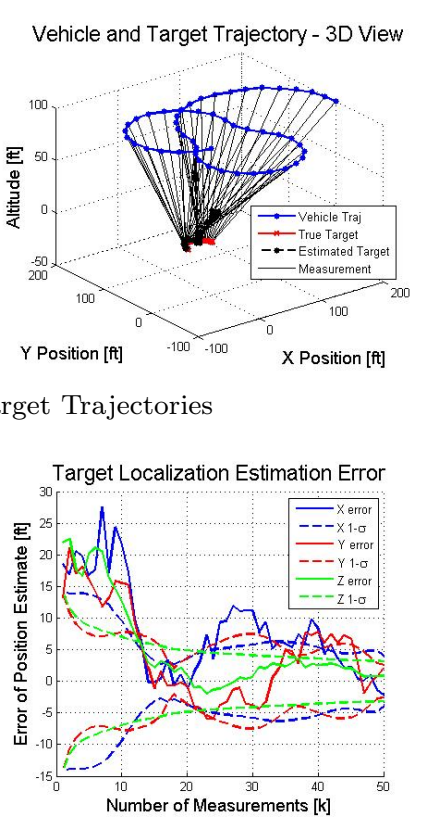

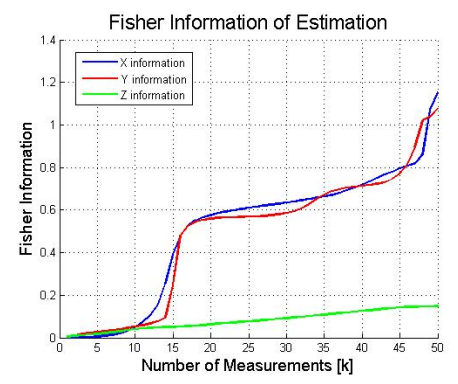

(b) Fisher Information

Figure 19: UAV trajectory optimization and EKF estimation results for a turning target

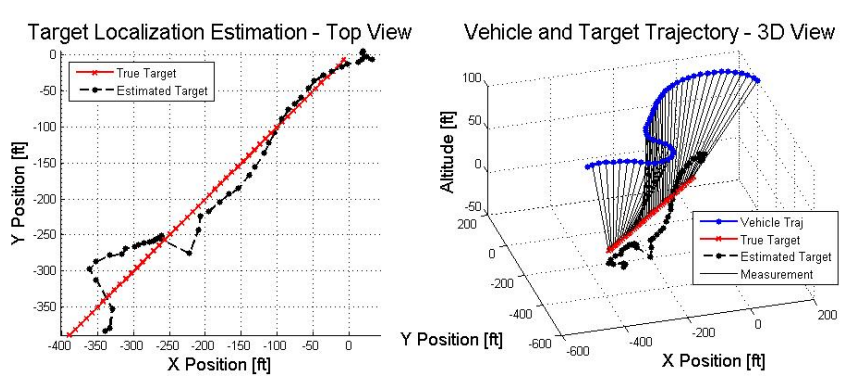

(a) Vehicle and Target Trajectories

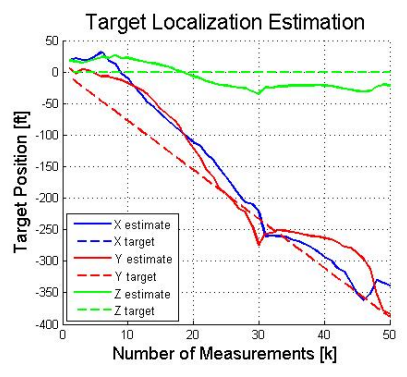

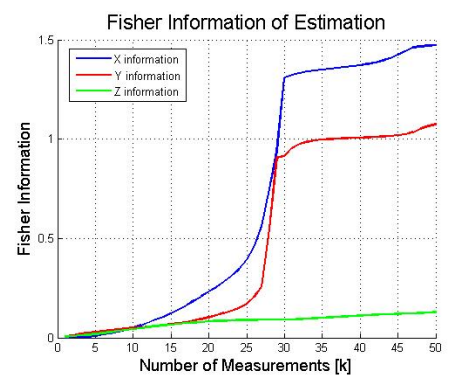

(b) Fisher Information

(c) Target Localization Estimation Results

Figure 20: UAV trajectory optimization and EKF estimation results for a fast constant velocity target 
0.5 seconds and, although computational resources available onboard a UAV are very different from those of a traditional personal computer, the algorithm run-time suggests that there is a future for implementing methods like these onboard small UAVs. Overall, the simultaneous estimation and trajectory optimization performs very well, showing that it is more important to increase the information provided by each new measurement by designing a proper UAV trajectory than to waste bandwidth and computational resources by taking more sub-optimal measurements, thus maximizing the amount of information provided by the measurements and increasing the performance of the target localization.

\section{Conclusions and Future Work}

This paper presents an information-theoretic approach for vehicle trajectory optimization in the 3-D bearings-only target localization problem. The Fisher Information Matrix and the Cramér-Rao Lower Bound are introduced as measures for assessing estimation performance and provide a framework for the development of vehicle trajectories that increase the information provided by the measurements in order to improve the accuracy and observability of the estimation. Optimization based on the FIM is shown to capture the essential geometric relationships associated with the estimation problem. Furthermore, the FIM is additive and separable providing a framework for processing measurements from various sensors and various timesteps using a simple summation, which makes the information-theoretic approach particularly well suited to estimation problems involving heterogenous sensors. For the 3-D bearings-only target localization problem the A-optimality criterion, involving the trace of the inverse of the FIM, is chosen as the best suited objective function. This function closely resembles the position dilution of precision (PDOP) criterion often used in GPS navigation, supporting the geometric intuition of the problem.

Optimal UAV trajectories are computed using gradient descent methods for different cases of target motion and vehicle constraints. For the sensor placement (unconstrained vehicle motion) case the optimal trajectory is shown to be a circle above the target with a radius to altitude ratio of approximately 0.7 . For the constrained vehicle motion scenario, constant velocity and maximum turn rate constraints are incorporated and the resulting trajectories consist of spirals that increase the angular separation between measurements while reducing the relative range to the target, thus supporting the geometric intuitions presented in Section I. As the turn rate constraint is relaxed, the UAV is free to make sharper turns and the resulting trajectories pass as close to the target as possible within the vehicle turn rate constraint. Sharper information increases are observed as the UAV passes closer to the target.

An Extended Kalman Filter (EKF) for the 3-D bearings-only target localization problem is presented and used to track stationary and moving targets from a fixed-wing small UAV platform. Simulation results for scenarios involving a predefined UAV trajectory are provided and compared to the cases using informationtheoretic trajectory optimization. The problem of simultaneous target estimation and vehicle trajectory optimization is explored and the resulting algorithms produce vehicle trajectories that increase the information provided by the measurements, enhancing the target estimation performance by increasing accuracy, reducing uncertainty and improving filter convergence. The total information obtained using trajectory optimization techniques is shown to be equal or greater with only half the measurements. Furthermore, the estimation accuracy is shown to increase and the uncertainty decrease when sharp information gains are obtained, showing the close relationship between the information and the estimation performance.

It is important to mention that the concepts of using the FIM to compute optimal trajectories for improved estimation performance, and combining the optimization and estimation, are not restricted to the bearingsonly target localization case. This information-theoretic approach provides a mathematical framework that can be used with any nonlinear system and any measurement model. Convergence properties may change based on the dynamics of the problem at hand but, since this approach relies on maximizing the information provided by the measurements and represents the underlying physical and geometric properties of the system, it is probable that these information-theoretic methods will enhance the estimation performance for many types of nonlinear systems.

Possible extensions of this work involve incorporating target motion models to improve estimation performance by increasing accuracy and filter stability. The trade-off is reduced flexibility, since deviations by the target from the expected behavior model would cause serious problems with the estimation. Some constraints could also be included, such as turn constraints or maximum vehicle speeds to limit the target's motion. Information about the environment could also be processed to produce probabilistic models of the target's motion (for example, if a vehicle is detected on a road, it is more likely to remain on the road than 
deviate off it). Research of target estimation using hospitability and synthetic inclination maps is already in place and could be used in combination with the trajectory optimization strategies provided in this work. Terrain elevation data, such as DTED, could also help refine the estimation of the $Z$ dimension. Other optimization considerations include environmental constraints such as obstacles or vehicle no-fly zones. Another extension of this project involves determining the optimal camera mounting location for a fixed camera. This camera placement would probably consist of a side-look camera since the optimal information-theoretic trajectory maintains a nearly orthogonal heading to the line-of-sight vector to the target. A consideration for small UAVs however, is that side-look cameras are more difficult to use since wind and gusts greatly affect the bank angle of the UAV. Another future work consideration involves optimizing the measurement rate, thus reducing processing requirements while still achieving the desired estimation accuracy. For example, the algorithm could be tuned to take a measurement only when the change in information is above a certain threshold, thus preserving bandwidth and computational resources.

\section{Acknowledgments}

The research leading to this article was partially supported by The Charles Stark Draper Laboratory, Inc, and by the Michigan/AFRL Collaborative Center on Control Sciences, AFOSR grant no. FA 8650-072-3744. Any opinions, findings, and conclusions or recommendations expressed in this publication are those of the authors and do not necessarily reflect the views of the supporting organizations.

\section{References}

${ }^{1}$ V. Aidala and S. Hammel. Utilization of modified polar coordinates for bearings-only tracking. Automatic Control, IEEE Transactions on, 28(3):283-294, Mar 1983.

${ }^{2}$ V. J. Aidala. Kalman filter behavior in bearings-only tracking applications. Aerospace and Electronic Systems, IEEE Transactions on, AES-15(1):29-39, Jan. 1979.

${ }^{3}$ V. J. Aidala and S. C. Nardone. Biased estimation properties of the pseudolinear tracking filter. Aerospace and Electronic Systems, IEEE Transactions on, AES-18(4):432-441, July 1982.

${ }^{4}$ Y. Bar-Shalom, T. Kirubarajan, and X.-R. Li. Estimation with Applications to Tracking and Navigation. John Wiley \& Sons, Inc., New York, NY, USA, 2002.

${ }^{5}$ D. B. Barber, J. Redding, T. Mclain, R. Beard, and C. Taylor. Vision-based target geo-location using a fixed-wing miniature air vehicle. J. Intell. Robotics Syst., 47(4):361-382, 2006.

${ }^{6} \mathrm{~K}$. Becker. Simple linear theory approach to tma observability. Aerospace and Electronic Systems, IEEE Transactions on, 29(2):575-578, Apr 1993.

${ }^{7}$ B. Bethke, M. Valenti, and J. How. Cooperative vision based estimation and tracking using multiple UAVs. In Conference of Cooperative Control and Optimization, Gainesville, FL, January 2007.

${ }^{8}$ W. G. Cochran. Experiments for nonlinear functions. Journal of the American Statistical Association, December 1973.

${ }^{9}$ J. H. de Vlieger and R. H. J. G. Meyling. Maximum likelihood estimation for long-range target tracking using passive sonar measurements. Signal Processing, IEEE Transactions on [see also Acoustics, Speech, and Signal Processing, IEEE Transactions on], 40(5):1216-1225, May 1992.

${ }^{10}$ J. A. Fawcett. Effect of course maneuvers on bearings-only range estimation. Acoustics, Speech, and Signal Processing [see also IEEE Transactions on Signal Processing], IEEE Transactions on, 36(8):1193-1199, Aug 1988.

${ }^{11}$ E. Fogel and M. Gavish. Nth-order dynamics target observability from angle measurements. Aerospace and Electronic Systems, IEEE Transactions on, 24(3):305-308, May 1988.

${ }^{12}$ E. Frew, C. Dixon, B. Argrow, and T. Brown. Radio source localization by a cooperating UAV team. In Infotech@Aerospace, Arlington, Virginia, September 2005.

${ }^{13}$ E. W. Frew. Trajectory Design for Target Motion Estimation Using Monocular Vision. PhD thesis, Stanford University, 2003.

${ }^{14}$ B. Grocholsky, A. Makarenko, and H. Durrant-Whyte. Information-theoretic control of multiple sensor platforms. In Proceedings of the IEEE International Conference on Robotics and Automation, Taipei, Taiwan, September 2003.

${ }^{15} \mathrm{~S}$. E. Hammel and V. J. Aidala. Observability requirements for three-dimensional tracking via angle measurements. Aerospace and Electronic Systems, IEEE Transactions on, AES-21(2):200-207, March 1985.

${ }^{16} \mathrm{~J}$. P. Helferty and D. R. Mudgett. Optimal observer trajectories for bearings only tracking by minimizing the trace of the cramer-rao lower bound. Decision and Control, 1993., Proceedings of the 32nd IEEE Conference on, pages 936-939 vol.1, 15-17 Dec 1993.

${ }^{17}$ J. P. Helferty, D. R. Mudgett, and J. E. Dzielski. Trajectory optimization for minimum range error in bearings-only source localization. OCEANS '93. 'Engineering in Harmony with Ocean'. Proceedings, pages II/229-II/234 vol.2, 18-21 Oct 1993.

${ }^{18}$ G. F. Ivey and E. N. Johnson. Investigation of methods for target state estimation using vision sensors. In $A I A A$ Guidance, Navigation, and Control Conference and Exhibit, San Francisco, CA, August 2005.

${ }^{19}$ L. M. Kaplan and V. Cevher. Design considerations for a heterogeneous network of bearings-only sensors using sensor management. Aerospace Conference, 2007 IEEE, pages 1-14, March 2007. 
${ }^{20}$ J. M. Kuang and M. Liu. Stereo vision based moving target tracking for omni-directional mobile robots in $2 \mathrm{~d}$ space. Information Acquisition, 2007. ICIA '07. International Conference on, pages 602-607, 8-11 July 2007.

${ }^{21}$ J. E. Le Cadre and C. Jauffret. Discrete-time observability and estimability analysis for bearings-only target motion analysis. Aerospace and Electronic Systems, IEEE Transactions on, 33(1):178-201, Jan. 1997.

${ }^{22}$ J. P. Le Cadre. Optimization of the observer motion for bearings-only target motion analysis. Decision and Control, 1997., Proceedings of the 36th IEEE Conference on, 4:3126-3131 vol.4, 10-12 Dec 1997.

${ }^{23}$ J. P. Le Cadre and H. Gauvrit. Optimization of the observer motion for bearings-only target motion analysis. Data Fusion Symposium, 1996. ADFS '96., First Australian, pages 190-195, 21-22 Nov 1996.

${ }^{24}$ E. L. Lehmann and G. Casella. Theory of Point Estimation. Springer Sciences+Business Media, LLC, New York, NY, 1998.

${ }^{25} \mathrm{~J}$. Levine and R. Marino. Constant-speed target tracking via bearings-only measurements. Aerospace and Electronic Systems, IEEE Transactions on, 28(1):174-182, Jan 1992.

${ }^{26}$ A. G. Lindgren and K. F. Gong. Position and velocity estimation via bearing observations. Aerospace and Electronic Systems, IEEE Transactions on, AES-14(4):564-577, July 1978.

${ }^{27} \mathrm{P}$. T. Liu. An optimum approach in target tracking with bearing measurements. Journal of Optimization Theory and Applications, 56(2):205-214, February 1988.

${ }^{28}$ A. Logothetis, A. Isaksson, and R. J. Evans. An information theoretic approach to observer path design for bearings-only tracking. Decision and Control, 1997., Proceedings of the 36th IEEE Conference on, 4:3132-3137 vol.4, Dec 1997.

${ }^{29} \mathrm{M}$. Mandic and E. Frazzoli. Efficient sensor coverage for acoustic localization. Decision and Control, 2007 46th IEEE Conference on, pages 3597-3602, Dec. 2007.

${ }^{30}$ S. Martínez and F. Bullo. Optimal sensor placement and motion coordination for target tracking. Automatica, 42(4):661668, April 2006.

${ }^{31}$ S. Nardone, A. Lindgren, and K. Gong. Fundamental properties and performance of conventional bearings-only target motion analysis. Automatic Control, IEEE Transactions on, 29(9):775-787, Sep 1984.

${ }^{32}$ S. C. Nardone and V. J. Aidala. Observability criteria for bearings-only target motion analysis. Aerospace and Electronic Systems, IEEE Transactions on, AES-17(2):162-166, March 1981.

${ }^{33}$ Y. Oshman and P. Davidson. Optimization of observer trajectories for bearings-only target localization. Aerospace and Electronic Systems, IEEE Transactions on, 35(3):892-902, Jul 1999.

${ }^{34}$ J. Ousingsawat and M. E. Campbell. Optimal cooperative reconnaissance using multiple vehicles. Journal Of Guidance, Control, and Dynamics, 30(1), 2007.

${ }^{35}$ J. M. Passerieux and D. Van Cappel. Optimal observer maneuver for bearings-only tracking. Aerospace and Electronic Systems, IEEE Transactions on, 34(3):777-788, Jul 1998.

${ }^{36}$ R. A. Pomranky. Human Robotics Interaction Army Technology Objective Raven Small Unmanned Aerial Vehicle Task Analysis and Modeling. Army Research Laboratory, January 2006.

${ }^{37}$ M. Quigley, M. A. Goodrich, S. Griffiths, A. Eldredge, and R. W. Beard. Target acquisition, localization, and surveillance using a fixed-wing mini-UAV and gimbaled camera. Robotics and Automation, 2005. ICRA 2005. Proceedings of the 2005 IEEE International Conference on, pages 2600-2605, April 2005.

${ }^{38}$ F. Rafi, S. Khan, K. Shafiq, and M. Shah. Autonomous target following by unmanned aerial vehicles. In Proceedings of SPIE, Orlando, FL, May 2006.

${ }^{39}$ B. Ristic, S. Arulampalam, and N. Gordon. Beyond the Kalman Filter: Particle Filters for Tracking Applications. Artech House, 2004

${ }^{40}$ A. Sinha, T. Kirubarajan, and Y. Bar-Shalom. Optimal cooperative placement of gmti UAVs for ground target tracking. Aerospace Conference, 2004. Proceedings. 2004 IEEE, 3:-1868 Vol.3, March 2004.

${ }^{41}$ X.-J. Tao, C.-R. Zou, and Z.-Y. He. Passive target tracking using maximum likelihood estimation. Aerospace and Electronic Systems, IEEE Transactions on, 32(4):1348-1354, Oct 1996.

$42 \mathrm{~J}$. H. Taylor. The cramer-rao estimation error lower bound computation for deterministic nonlinear systems. Decision and Control including the 17th Symposium on Adaptive Processes, 1978 IEEE Conference on, 17:1178-1181, Jan. 1978.

${ }^{43}$ P. Tichavsky, C. H. Muravchik, and A. Nehorai. Posterior cramer-rao bounds for discrete-time nonlinear filtering. Signal Processing, IEEE Transactions on [see also Acoustics, Speech, and Signal Processing, IEEE Transactions on], 46(5):1386-1396, May 1998.

${ }^{44}$ D. Uciński. Optimal Measurement Methods for Distributed Parameter System Identification. CRC Press, 2005.

${ }^{45}$ Y. Watanabe, E. N. Johnson, and A. J. Calise. Optimal 3-d guidance from a 2-d vision sensor. AIAA Guidance, Navigation, and Control Conference, August 2004.

${ }^{46} \mathrm{~W}$. Whitacre and M. Campbell. Information-theoretic optimization of periodic orbits for cooperative geolocation. Technical report, Cornell University, Ithaca, NY, 2007.

${ }^{47}$ R. A. Wise and R. T. Rysdyk. UAV coordination for autonomous target tracking. In AIAA Guidance, Navigation, and Control Conference and Exhibit, Keystone, CO, August 2006. 